\title{
Characterization of moose intestinal glycosphingolipids
}

\author{
Miralda Madar Johansson ${ }^{1} \cdot$ Benjamin Dedic $^{1} \cdot$ Klara Lundholm $^{1}$ • \\ Filip Berner Branzell ${ }^{1}$ • Angela Barone ${ }^{1}$. John Benktander ${ }^{1} \cdot$ Susann Teneberg ${ }^{1}$
}

Received: 4 May 2015 / Revised: 28 May 2015 / Accepted: 3 June 2015 /Published online: 24 June 2015

(C) The Author(s) 2015. This article is published with open access at Springerlink.com

\begin{abstract}
As a part of a systematic investigation of the species-specific expression of glycosphingolipids, acid and non-acid glycosphingolipids were isolated from three small intestines and one large intestine of the moose (Alces alces). The glycosphingolipids were characterized by binding of monoclonal antibodies, lectins and bacteria in chromatogram binding assays, and by mass spectrometry. The non-acid fractions were complex mixtures, and all had glycosphingolipids belonging to the lacto- and neolactoseries (lactotriaosylceramide, lactotetraosylceramide, neolactotetraosylceramide, Gal $\alpha 3-\mathrm{Le}^{\mathrm{x}}$ hexaosylceramide, and lacto-neolactohexaosylceramide), globoseries (globotriaosylceramide and globotetraosylceramide), and isogloboseries (isoglobotriaosylceramide). Penta- and heptaglycosylceramides with terminal Galili determinants were also characterized. Furthermore, glycosphingolipids with terminal blood group $\mathrm{O}$ determinants ( $\mathrm{H}$ triaosylceramide, $\mathrm{H}$ type 2 pentaosylceramide, $\mathrm{H}$ type 1 penta- and heptaosylceramide) were characterized in two of the moose small intestines, and in the one large intestine, while the third small intestine had glycosphingolipids with terminal blood group A determinants (A tetraosylceramide, A type 1 hexa- and octaosylceramide, A dodecaosylceramide). The acid glycosphingolipid fractions of moose small and large intestine contained sulfatide, and the
\end{abstract}

Electronic supplementary material The online version of this article (doi:10.1007/s10719-015-9604-8) contains supplementary material, which is available to authorized users.

Susann Teneberg

Susann.Teneberg@medkem.gu.se

Institute of Biomedicine, Department of Medical Biochemistry and Cell Biology, University of Gothenburg, P.O. Box 440, S-405 30 Gothenburg, Sweden gangliosides GM3, GD3, GD1a, GD1b, and also NeuGc and NeuAc variants of the $\mathrm{Sd}^{\mathrm{a}}$ ganglioside and the sialylglobopenta/SSEA-4 ganglioside. In humans, the NeuAcglobopenta/SSEA-4 ganglioside is a marker of embryonic and adult stem cells, and is also expressed in several human cancers. This is the first time sialyl-globopentaosylceramide/ SSEA-4 has been characterized in a fully differentiated normal tissue, and also the first time NeuGc-globopentaosylceramide has been characterized.

Keywords Glycosphingolipid characterization · Mass spectrometry $\cdot$ Blood group antigens $\cdot \mathrm{SSEA}-4 \cdot \mathrm{Sd}^{\mathrm{a}}$ determinant
Abbreviations
LC-ESI/MS Liquid chromatography/electrospray ionization mass spectrometry
SSEA Stage specific embryonic antigen

\section{Introduction}

Glycosphingolipids are present in all vertebrate cell membranes and, due to their strategic position on the outer membrane, they can participate in a large number of recognition processes. The expression of glycosphingolipids differs between species, individuals, tissues and cells, and there are also developmental changes. This variable expression among species may be illustrated by the non-acid glycosphingolipids of the small intestine, where the mouse has mainly ganglio series compounds, the dog, cat, and rabbit mainly glycosphingolipids with neolacto/type 2 core, and the predominant complex glycosphingolipids of 
human, porcine and rat small intestine are based on lacto/type 1 core chains [1-3].

So far mainly the intestinal glycosphingolipids of domestic and laboratory animals have been characterized. In order to broaden the perspective, we have in this study isolated acid and non-acid glycosphingolipids from three small intestines and one large intestine of the moose (Alces alces). The glycosphingolipids were characterized by binding of monoclonal antibodies, lectins and bacteria in chromatogram binding assays, and by mass spectrometry. As in the human small intestine, the complex non-acid glycosphingolipids had mainly lacto/type 1 core chains. Glycosphingolipids with blood group A determinants were characterized in one of the small intestinal samples, while blood group $\mathrm{H}$ determinants were present in the non-acid glycosphingolipid fractions of the other two moose small intestines, and the large intestinal fraction. The acid fractions contained sulfatide and a complex mixture of NeuGc- and NeuAc-carrying gangliosides. Here, NeuGc and NeuAc variants of the sialyl-globopenta/ SSEA-4 ganglioside and the $\mathrm{Sd}^{\mathrm{a}}$ ganglioside were characterized, in addition to the more "ordinary" gangliosides as GM3, GD3, GD1a and GD1b.

\section{Materials and methods}

\section{Glycosphingolipid preparations}

The intestines from the three moose were gifts from the local hunters. After the animals were killed the intestines were taken out and washed carefully in a small river. The material was kept on ice until delivery at the laboratory, where it was frozen at $-70^{\circ} \mathrm{C}$. The intestines were then lyophilized, and acid and non-acid glycosphingolipids were thereafter isolated as described [4]. Thus, the intestines were extracted in two steps in a Soxhlet apparatus with chloroform and methanol (2:1 and $1: 9$, by volume, respectively). The material thereby obtained was subjected to mild alkaline hydrolysis and dialysis, followed by separation on a silicic acid column. Acid and non-acid glycosphingolipid fractions were obtained by chromatography on a DEAE-cellulose column. In order to separate the non-acid glycosphingolipids from alkali-stable phospholipids, the non-acid fraction was acetylated and separated on a second silicic acid column, followed by deacetylation and dialysis. Final purifications were done by chromatographies on DEAE-cellulose and silicic acid columns.

Thereafter, $80 \mathrm{mg}$ of the total non-acid glycosphingolipid fraction from moose I small intestine was separated on a silicic acid column eluted with increasing volumes of methanol in chloroform. Thereby one fraction containing monoglycosylceramides (denoted fraction N-I) and one fraction containing diglycosylceramides and more slow-migrating glycosphingolipids was obtained. The latter fraction $(32.0 \mathrm{mg})$ was separated on an Iatrobeads (Iatrobeads 6RS-8060; Iatron Laboratories, Tokyo) column (10 g) eluted with increasing volumes of methanol in chloroform. This gave one monoand diglycosylceramide fraction $(7.4 \mathrm{mg}$; fraction $\mathrm{N}-\mathrm{II})$, and one fraction containing triglycosylceramides and more slowmigrating glycosphingolipids $(19.0 \mathrm{mg})$. This fraction was separated on an Iatrobeads column (25 g) eluted with $60 \times 1 \mathrm{ml}$ chloroform/methanol/water 60:35:8 (by volume), followed by $10 \mathrm{ml}$ chloroform/methanol/water $60: 35: 8$, $20 \mathrm{ml}$ chloroform/methanol/water $60: 35: 8$ and finally $50 \mathrm{ml}$ chloroform/methanol/water 60:35:8. Throughout the separation procedures aliquots of the fractions obtained were analyzed by thin-layer chromatography and anisaldehyde detection. The fractions were pooled according to their migration on thin-layer chromatograms giving five fractions denoted $\mathrm{N}$ III (0.5 mg), N-IV (7.9 mg), N-V (3.6 mg), N-VI (3.0 mg), and N-VII (0.9 mg), respectively.

The non-acid glycosphingolipids of moose II small intestine were also separated by repeated chromatographies on Iatrobeads columns. Here we focused on isolation of compounds recognized by antibodies directed against the blood group A determinant. Thus, the fractions were pooled according to their migration on thin-layer chromatograms and their reactivity with the monoclonal anti-A antibodies. Finally, ten subfractions were collected (denoted fractions N2-I - N2-X). Fraction N2-IV had binding of anti-A antibodies in the tetraosylceramide region, fraction N2-VII had anti-A binding in the hexa- and octaosylceramide regions, while in fraction N2-IX the antibodies bound in octaosylceramide region and to a slow-migrating compound.

A similar strategy was used for separation of the total acid glycosphingolipid fractions from moose I small intestine. Here the acid glycosphingolipids (160 mg) were first separated on an Iatrobeads column ( $2 \mathrm{~g})$ eluted with increasing volumes of methanol in chloroform. The glycosphingolipid fraction obtained $(88.3 \mathrm{mg}$ ) was again separated on an Iatrobeads column ( $1 \mathrm{~g}$ ) eluted with increasing volumes of methanol in chloroform. This gave one fraction (15.3 mg denoted fraction A-I) co-migrating with sulfatide on thin-layer chromatograms, one fraction (30.5 mg; fraction A-II) co-migrating with the GM3 ganglioside, and one fraction (20.6 mg) containing slow-migrating gangliosides. The latter fraction was separated on three subsequent Iatrobeads columns eluted with chloroform/methanol/water 60:35:8 (by volume). Aliquots of the fractions obtained were analyzed by thin-layer chromatography and detection with anisaldehyde and resorcinol. The fractions were pooled according to their migration on thinlayer chromatograms giving five fractions denoted A-III (0.5 mg), A-IV (1.1 mg), A-V (0.4 mg), A-VI (1.8 mg), and A-VII $(0.5 \mathrm{mg})$, respectively.

Separation of the total acid and the total non-acid glycosphingolipid fractions from moose I large intestine was done in the same manner. Thus, the total acid or non-acid 
glycosphingolipid fractions were first separated on a silicic acid column eluted with increasing volumes of methanol in chloroform. This was followed by repeated chromatographies on Iatrobeads columns eluted with chloroform/methanol/ water 60:35:8 (by volume).

\section{Reference glycosphingolipids}

Total acid and non-acid glycosphingolipid fractions were isolated as described [4]. Individual glycosphingolipids were isolated by repeated chromatography on silicic acid columns and by HPLC, and identified by mass spectrometry $\left([5,6]\right.$ and ${ }^{1} \mathrm{H}-$ NMR spectroscopy [7].

\section{Thin-layer chromatography}

Thin-layer chromatography was done on aluminum- or glassbacked silica gel 60 high performance thin-layer chromatography plates (Merck, Darmstadt, Germany). Glycosphingolipid mixtures $(20-40 \mu \mathrm{g})$ or pure glycosphingolipids $(4 \mu \mathrm{g})$ were applied to the plates, and eluted with chloroform/methanol/ water (60:35:8, by volume) as solvent system. Chemical detection was done with anisaldehyde [8], or the resorcinol reagent [9].

\section{Chromatogram binding assays}

Erythrina cristagalli lectin was purchased from Vector Labs, and Griffonia simplicifolia IB4 lectin from Advanced Targeting Systems. Monoclonal anti-A (HE193), anti-B (HEB-29), and anti-H type 1 (17-206) antibodies were obtained from GeneTex/Abcam. Rabbit anti-mouse antibodies (Z0259) were from DakoCytomation Norden A/S, anti-globopenta/ SSEA-3 (MC-631), and anti-sialyl-globopenta/SSEA-4 (MC813-70) from eBioscience, anti-GD1a (GD1a-1) from Millipore, and anti-Lewis ${ }^{\mathrm{x}}(\mathrm{P} 12)$ was from Calbiochem. Rabbit polyclonal anti-GM2 serum was purchased from Calbiochem, and goat anti-rabbit serum was from Aviva Systems Biology.

Binding of antibodies to glycosphingolipids on thin-layer chromatograms was done as described previously [10]. Dried chromatograms were dipped in diethylether $/ n$-hexane $(1: 5 v / v)$ containing $0.5 \%(w / v)$ polyisobutylmethacrylate for $1 \mathrm{~min}$. To diminish background binding the chromatograms were blocked for $2 \mathrm{~h}$ at room temperature with phosphatebuffered saline (PBS, $\mathrm{pH} 7.3)$ containing $2 \%(w / v)$ bovine serum albumin and $0.1 \%(w / v) \mathrm{NaN}_{3}$ (BSA/PBS). Suspensions of monoclonal antibodies diluted in BSA/PBS (the dilution used for each antibody is given in Table 1) were gently sprinkled over the chromatograms, followed by incubation for $2 \mathrm{~h}$ at room temperature. After washing with PBS followed a second $2 \mathrm{~h}$ incubation with ${ }^{125}$ I-labeled (labeled according to the IODO-GEN protocol of the manufacturer (Pierce, Rockford, IL)) rabbit anti-mouse antibodies, or goat anti-rabbit antibodies, diluted to $2 \times 10^{6} \mathrm{cpm} / \mathrm{ml}$ in BSA/PBS. Finally, the plates were washed six times with PBS. Dried chromatograms were autoradiographed for $12-72 \mathrm{~h}$ using XAR-5 X-ray films (Eastman Kodak).

Chromatogram binding assays with ${ }^{125}$ I-labeled E. cristagalli lectin, and G. simplicifolia IB4 lectin, were done as described [11]. Binding of ${ }^{35} \mathrm{~S}$-labeled BabA expressing H. pylori strain J99 to glycosphingolipids on thin-layer chromatograms was done as described [12].

\section{Endoglycoceramidase digestion and LC-ESI/MS of oligosaccharides}

Endoglycoceramidase II from Rhodococcus spp. (Takara Bio Europe S.A., Gennevilliers, France) was used for hydrolysis. Briefly, $50 \mu \mathrm{g}$ of non-acid glycosphingolipids were resuspended in $100 \mu 10.05 \mathrm{M}$ sodium acetate buffer, $\mathrm{pH}$ 5.0, containing $120 \mu \mathrm{g}$ sodium cholate, and sonicated briefly. Thereafter, $1 \mathrm{mU}$ of endoglycoceramidase II was added and the mixture was incubated at $37^{\circ} \mathrm{C}$ for $48 \mathrm{~h}$. The reaction was stopped by addition of chloroform/methanol/water to the final proportions 8:4:3 (by volume). The oligosaccharidecontaining upper phase thus obtained was separated from detergent on a Sep-Pak QMA cartridge (Waters, Milford, MA). The eluant containing the oligosaccharides was dried under nitrogen and under vacuum.

The glycosphingolipid-derived oligosaccharides were resuspended in $50 \mu \mathrm{l}$ of water and analyzed by LC-ESI/MS as described [6]. The oligosaccharides were separated on a column $(200 \times 0.180 \mathrm{~mm})$ packed in-house with $5 \mu \mathrm{m}$ porous graphite particles (Hypercarb, Thermo-Hypersil, Runcorn, UK). An autosampler, HTC-PAL (CTC Analytics AG, Zwingen, Switzerland) equipped with a cheminert valve ( $0.25 \mathrm{~mm}$ bore) and a $2 \mu \mathrm{l}$ loop, was used for sample injection. An Agilent 1100 binary pump (Agilent technologies, Palo Alto, CA) delivered a flow of $250 \mu \mathrm{l} / \mathrm{min}$, which was splitted down in an 1/16" microvolume-T ( $0.15 \mathrm{~mm}$ bore) (Vici AG International, Schenkon, Switzerland) by a $50 \mathrm{~cm} \times 50 \mu \mathrm{m}$ i.d. fused silica capillary before the injector of the autosampler, allowing approximately $2-3 \mu \mathrm{l} / \mathrm{min}$ through the column. The oligosaccharides $(3 \mu \mathrm{l})$ were injected on to the column and eluted with an acetonitrile gradient (A: $10 \mathrm{mM}$ ammonium bicarbonate; B: $10 \mathrm{mM}$ ammonium bicarbonate in $80 \%$ acetonitrile). The gradient $(0-45 \% \mathrm{~B})$ was eluted for $46 \mathrm{~min}$, followed by a wash step with $100 \% \mathrm{~B}$, and equilibration of the column for $24 \mathrm{~min}$. A $30 \mathrm{~cm} \times 50 \mu \mathrm{m}$ m i.d. fused silica capillary was used as transfer line to the ion source.

The saccharides were analyzed in negative ion mode on an LTQ linear quadrupole ion trap mass spectrometer (Thermo Electron, San José, CA). The IonMax standard ESI source on the LTQ mass spectrometer was equipped with a stainless steel needle kept at $-3.5 \mathrm{kV}$. Compressed air was used as nebulizer gas. The heated capillary was kept at $270{ }^{\circ} \mathrm{C}$, and the capillary 
Table 1 Antibodies used in chromatogram binding assays

\begin{tabular}{lllll}
\hline Antibodies & Clone & Manufacturer & Dilution & Isotype \\
\hline Anti-blood group A & HE-193 & GeneTex/Abcam & $1: 500$ & IgM \\
Anti-blood group B & HEB-29 & GeneTex/Abcam & $1: 100$ & IgM \\
Anti-blood group H type 1 & $17-206$ & GeneTex/Abcam & $1: 100$ & IgG3 \\
Anti-Globopenta/SSEA-3 & MC-631 & eBioscience & $1: 50$ & IgM \\
Anti-Lewix $/$ SSEA-1 & P12 & Calbiochem & $1: 200$ & IgM \\
Anti-Sialyl-globopenta/SSEA-4 & MC-813-70 & eBioscience & $1: 100$ & IgG3 \\
Anti-GD1a & GD1a-1 & Millipore & $1: 100$ & IgG1 \\
Anti-GM2 (polyclonal) & - & Calbiochem & $1: 100$ & \\
\hline
\end{tabular}

voltage was $-50 \mathrm{kV}$. Full-scan ( $\mathrm{m} / \mathrm{z} 380-2$ 000, 2 microscans, maximum $100 \mathrm{~ms}$, target value of 30 000) was performed, followed by data dependent $\mathrm{MS}^{2}$ scans of the three most abundant ions in each scan (2 microscans, maximum $100 \mathrm{~ms}$, target value of 10000$)$. The threshold for $\mathrm{MS}^{2}$ was set to 500 counts. Normalized collision energy was $35 \%$, and an isolation window of $3 \mathrm{u}$, an activation $\mathrm{q}=0.25$, and an activation time of $30 \mathrm{~ms}$, was used. Data acquisition and processing were conducted with Xcalibur software (Version 2.0.7). Manual assignment of glycan sequences was done on the basis of knowledge of mammalian biosynthetic pathways, with the assistance of the Glycoworkbench tool (Version 2.1), and by comparison of retention times and $\mathrm{MS}^{2}$ spectra of oligosaccharides from reference glycosphingolipids [6].

\section{LC-ESI/MS of native glycosphingolipids}

Glycosphingolipids were dissolved in methanol:acetonitrile in proportion 75:25 (by volume) and separated on a $200 \times$ $0.150 \mathrm{~mm}$ column, packed in-house with $5 \mu \mathrm{M}$ polyamine II particles (YMC Europe GmbH, Dinslaken, Germany). An autosampler, HTC-PAL equipped with a cheminert valve ( $0.25 \mathrm{~mm}$ bore) and a $2 \mu \mathrm{lloop}$, was used for sample injection. An Agilent 1100 binary pump delivered a flow of $250 \mu \mathrm{l} / \mathrm{min}$, which was splitted down in an 1/16" microvolume-T ( $0.15 \mathrm{~mm}$ bore) by a $50 \mathrm{~cm} \times 50 \mu \mathrm{m}$ i.d. fused silica capillary before the injector of the autosampler, allowing approximately 2-3 $\mu \mathrm{l} / \mathrm{min}$ through the column. Samples were eluted with an aqueous gradient (A:100\% acetonitrile to B: $10 \mathrm{mM}$ ammonium bicarbonate). The gradient $(0-50 \% \mathrm{~B})$ was eluted for $40 \mathrm{~min}$, followed by a wash step with $100 \% \mathrm{~B}$, and equilibration of the column for $20 \mathrm{~min}$. The samples were analyzed in negative ion mode on a LTQ linear quadropole ion trap mass spectrometer, with an IonMax standard ESI source equipped with a stainless steel needle kept at $-3.5 \mathrm{kV}$. Compressed air was used as nebulizer gas. The heated capillary was kept at $270{ }^{\circ} \mathrm{C}$, and the capillary voltage was $-50 \mathrm{kV}$. Full scan ( $m / z$ 500-1800, two microscan, maximum $100 \mathrm{~ms}$, target value of 30,000) was performed, followed by data-dependent $\mathrm{MS}^{2}$ scans (two microscans, maximun $100 \mathrm{~ms}$, target value of $10.000)$ with normalized collision energy of $35 \%$, isolation window of 2.5 units, activation $\mathrm{q}=0.25$ and activation time $30 \mathrm{~ms}$ ). The threshold for $\mathrm{MS}^{2}$ was set to 500 counts. Data acquisition and processing were conducted with Xcalibur software (Version 2.0.7). Manual assignment of glycosphingolipid sequences was done with the assistance of the Glycoworkbench tool (Version 2.1), and by comparison of retention times and $\mathrm{MS}^{2}$ spectra of reference glycosphingolipids.

\section{Results}

\section{Isolation of glycosphingolipids from moose intestines}

Total acid and non-acid glycosphingolipids (exemplified in Fig. 1) were isolated from moose small and large intestines by standard procedures [4]. The acid and non-acid glycosphingolipid fractions from moose I and II small intestines, and moose I large intestine, were separated by chromatographies on silicic acid and Iatrobodies columns. In the case of moose I small intestine, this gave seven non-acid and seven acid subfractions. The non-acid subfractions contained compounds migrating as monoglycosylceramides (fraction N-I; not shown), mono- and diglycosylceramides (fraction NII), di- and triglycosylceramides (fraction N-III), tri- and tetraglycosylceramides (fraction N-IV), tetraglycosylceramides (fraction $\mathrm{N}-\mathrm{V}$ ), pentaglycosylceramides (fraction N-VI), and heptaglycosylceramides (fraction N-VII) (Fig. 2a).

In the case of moose II small intestine ten subfractions were collected (denoted fractions N2-I-N2-X).

The seven acid subfractions from moose I small intestine are shown in Fig. $2 b$ and c. The main compound of fraction A-I migrated relatively high on the thin-layer chromatogram, and was not stained by resorcinol. The other six fractions were resorcinol positive, indicating sialic acid-containing glycosphingolipids i.e., gangliosides. Fraction A-II migrated at the level of the GM3 ganglioside, while the gangliosides of fraction A-III- A-VI were more slow-migrating. 


\section{Anisaldehyde detection}

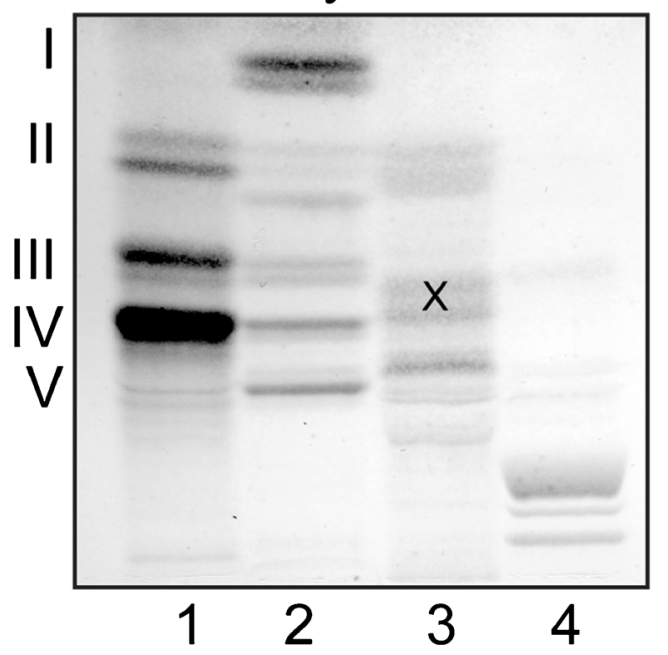

Fig. 1 Thin-layer chromatogram of glycosphingolipids isolated from moose I small intestine. The chromatograms were eluted with chloroform/methanol/water 60:35:8 (by volume), and detection of glycosphingolipids was done with anisaldehyde. The lanes were: Lane 1 , non-acid glycosphingolipids of human blood group $\mathrm{AB}$ erythrocytes, $40 \mu \mathrm{g}$; Lane 2, total non-acid glycosphingolipids of moose I small intestine, $40 \mu \mathrm{g}$; Lane 3, total acid glycosphingolipids of moose I small intestine, $40 \mu \mathrm{g}$; Lane 4 , calf brain gangliosides, $40 \mu \mathrm{g}$. The Roman numbers to the left of panel A indicate the approximate number of carbohydrate units in the bands. The bands marked with an $\mathrm{X}$ in lane 3 are non-glycosphingolipid contaminants

\section{Characterization of the non-acid glycosphingolipids of moose I small intestine}

Chromatogram binding assays At first, the binding of a number of carbohydrate binding ligands to the non-acid glycosphingolipids from moose intestines was tested. The Gal $\alpha 3$ binding IB4 lectin from G. simplicifolia recognized compounds migrating in the penta- and heptaglycosylceramide regions in the non-acid fractions from the three moose small intestines and the one moose large intestine (Fig. 3b, lanes 2-5). The lectin from E. cristagalli binds to glycoconjugates with terminal Gal $\beta 4 \mathrm{GlcNAc}$ and Fuc $\alpha 2$ Gal $\beta 4$ GlcNAc sequences [11]. Binding of the E. cristagalli lectin in the tetraglycosylceramide region in the non-acid fractions from moose small and large intestines was obtained (Fig. 3c, lanes 2-5), indicating the presence of a Gal 34 GlcNAc-terminated glycosphingolipid, i.e., neolactotetraosylceramide ( $\mathrm{Gal} \beta 4 \mathrm{GlcNAc} \beta 3 \mathrm{Gal} \beta 4 \mathrm{Glc} \beta 1 \mathrm{Cer})$. The monoclonal antibodies directed against the blood group $\mathrm{H}$ type 1 epitope bound to compounds migrating in the penta- and heptaglycosylceramide regions in the non-acid fractions from the small intestine of moose I and III, and the large intestine of moose I (Fig. 3d, lanes 2, 4 and 5), indicating the presence of Fuc $\alpha 2 \mathrm{Gal} \beta 3 \mathrm{GlcNAc}$-terminated penta- and heptaglycosylceramides. However, no binding of the anti-H type 1 antibodies to the non-acid glycosphingolipid

\section{a Anisaldehyde detection}

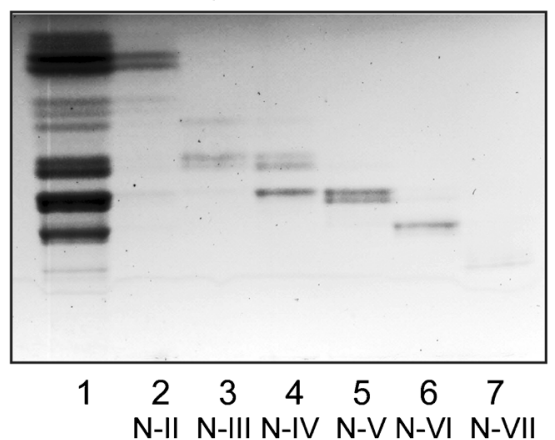

b Anisaldehyde detection

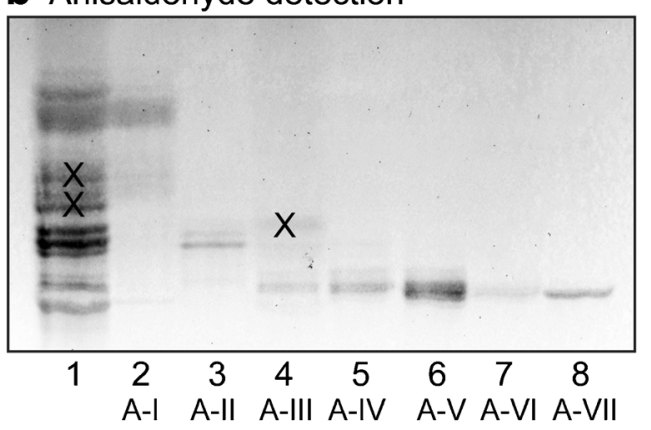

\section{Resorcinol detection}

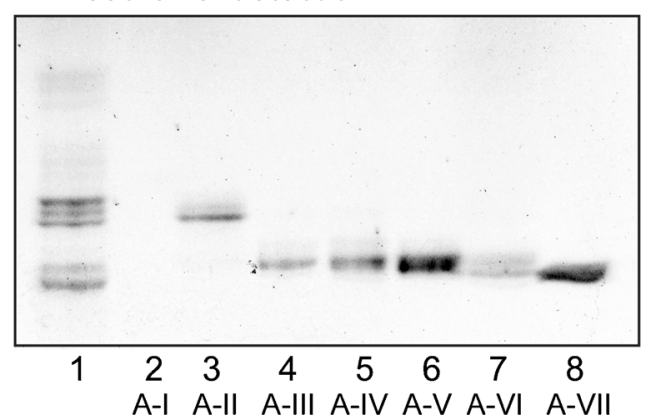

Fig. 2 Separation of glycosphingolipids from moose I small intestine. Thin-layer chromatogram of non-acid (a) and acid (b and c) glycosphingolipid fractions isolated from moose I small intestine. The chromatograms were eluted with chloroform/methanol/water 60:35:8 (by volume), and detection of glycosphingolipids was done with anisaldehyde (a and $\mathbf{b}$ ), and the resorcinol reagent (c). The lanes on (a) were: Lane 1, total non-acid glycosphingolipids of moose I small intestine, $40 \mu \mathrm{g}$; Lanes 2-7, fractions N-II - N-VII from moose I small intestine, $4 \mu \mathrm{g} /$ lane. The lanes on (b and $\mathbf{c}$ ) were: Lane 1, total acid glycosphingolipids of moose I small intestine, $40 \mu \mathrm{g}$; Lanes 2-8, fraction A-I - A-VII from moose I small intestine, $4 \mu \mathrm{g} /$ lane. The bands marked with $\mathrm{X}$ on (b) are non-glycosphingolipid contaminants

fraction of moose II small intestine was obtained (Fig. 3d, lane 3). Instead, in moose II small intestine fraction there was a distinct binding of monoclonal antibodies directed against blood group A determinant to compounds migrating as tetraand hexaglycosylceramides, and also more slow-migrating glycosphingolipids (Fig. 3e, lane 3). The BabA adhesin of Helicobacter pylori strain $\mathrm{J} 99$ recognizes blood group A/B/ $\mathrm{H}$ determinants on type 1 and type 4 core chains, in addition to 
Fig. 3 Comparison of the nonacid glycosphingolipid fractions from moose small and large intestine. Thin-layer chromatogram after detection with anisaldehyde (a), and autoradiograms obtained by binding of G. simplicifolia IB4 lectin (b), E. cristagalli lectin (c), the monoclonal anti-H type 1 antibody 17-206 (d), the monoclonal anti-A antibody HE193 (e), and BabA expressing Helicobacter pylori strain $\mathrm{J} 99$ (f). The glycosphingolipids were separated on aluminum-backed silica gel plates, using chloroform/methanol/water 60:35:8 (by volume) as solvent system, and the binding assays were performed as described under "Materials and methods". Autoradiography was for $12 \mathrm{~h}$. The lanes were: Lane 1, total nonacid glycosphingolipids of human blood group $\mathrm{AB}$ erythrocytes, $40 \mu \mathrm{g}$; Lane 2, total non-acid glycosphingolipids of moose I small intestine, $40 \mu \mathrm{g}$; Lane 3 , total non-acid glycosphingolipids of moose II small intestine, $40 \mu \mathrm{g}$; Lane 4, total non-acid glycosphingolipids of moose III small intestine, $40 \mu \mathrm{g}$; Lane 5 , total non-acid glycosphingolipids of moose I large intestine, $40 \mu \mathrm{g}$ a Anisaldehyde detection

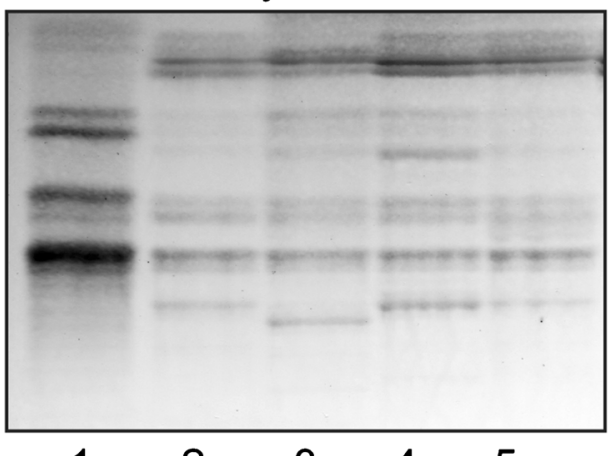

$\begin{array}{lllll}1 & 2 & 3 & 4 & 5\end{array}$

c E. cristagalli lectin

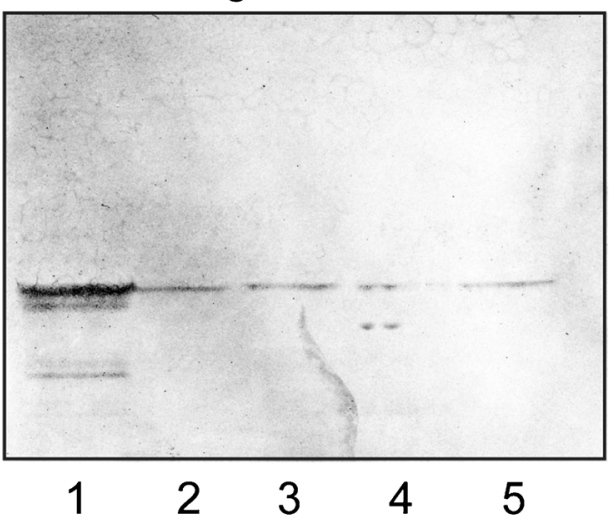

e Anti-A

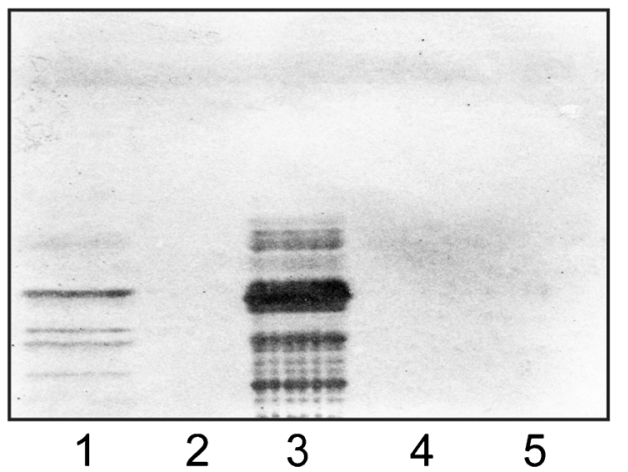

b G. simplicifolia IB4 lectin

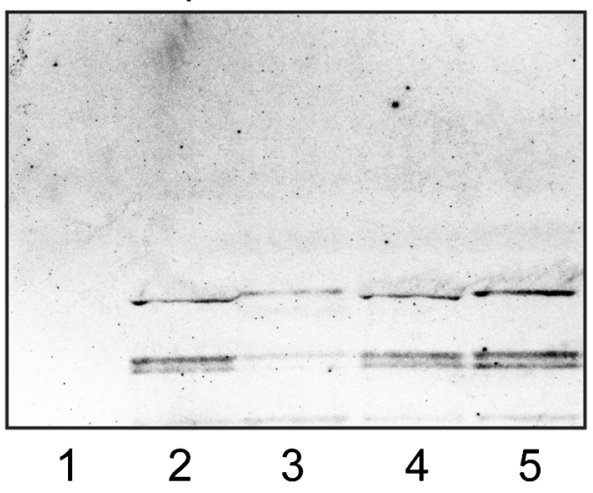

d Anti-H type 1

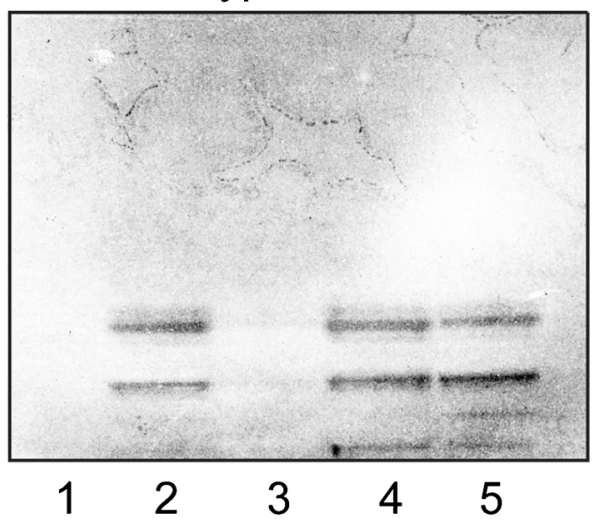

f Helicobacter pylori $\mathrm{J99}$

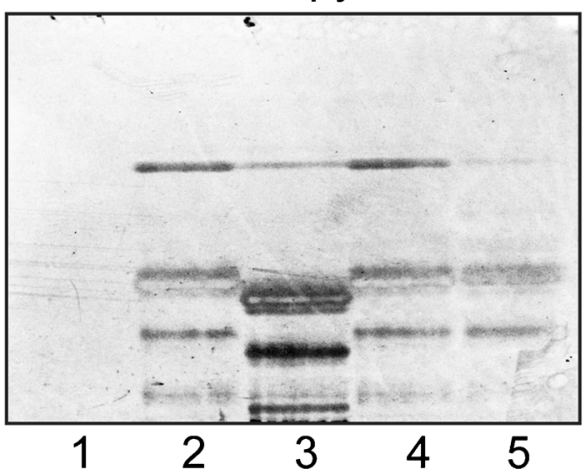

binding to the $\mathrm{Le}^{\mathrm{b}}$ determinant [12]. Upon binding of the J99 strain to the moose intestinal glycosphingolipids, the binding pattern obtained appeared as a merge of the binding obtained with the anti-H type 1 antibodies and the anti-A antibodies (Fig. 3f, lanes 2-5). In addition there was a binding in the diglycosylceramide region, most likely representing $H$. pylori lactosylceramide binding [13].

Binding of monoclonal antibodies directed against the blood group B and the globopenta/SSEA-3 epitopes to the non-acid glycosphingolipids from moose intestines was also tested. However, no binding was observed (data not shown).
Next, the binding of lectins and monoclonal antibodies to the non-acid subfractions isolated from the small intestine of moose I was evaluated. Here the E. cristagalli lectin bound to fraction $\mathrm{N}-\mathrm{V}$ (Fig. 4b), while the anti $\mathrm{H}$ type 1 binding activity was found in fractions N-V and N-VI (pentaglycosylceramide region), and fraction N-VII (heptaglycosylceramide region) (Fig. 4c). Binding to fraction N-V I (pentaglycosylceramide region), and fraction N-VII (heptaglycosylceramide region) was also obtained with the G. simplicifolia IB4 lectin (Fig. 4d). A weak but distinct 
Fig. 4 Binding of lectins and monoclonal antibodies to the nonacid glycosphingolipid subfractions isolated from moose I small intestine. Thin-layer chromatogram after detection with anisaldehyde (a), and autoradiograms obtained by binding of E. cristagalli lectin (b), the monoclonal anti-H type 1 antibody 17-206 (c),

G. simplicifolia IB4 lectin (d), and the monoclonal anti-Lewis ${ }^{\mathrm{x}}$ antibody P12 (e). The glycosphingolipids were separated on aluminum-backed silica gel plates, using chloroform/methanol/water 60:35:8 (by volume) as solvent system, and the binding assays were performed as described under "Materials and methods". Autoradiography was for $12 \mathrm{~h}$. The lanes were: Lane 1, total nonacid glycosphingolipids of moose I small intestine, $40 \mu \mathrm{g}$; Lanes 25 , fraction N-IV - N-VII from moose I small intestine, $4 \mu \mathrm{g} / \mathrm{lane}$

\section{a Anisaldehyde detection}

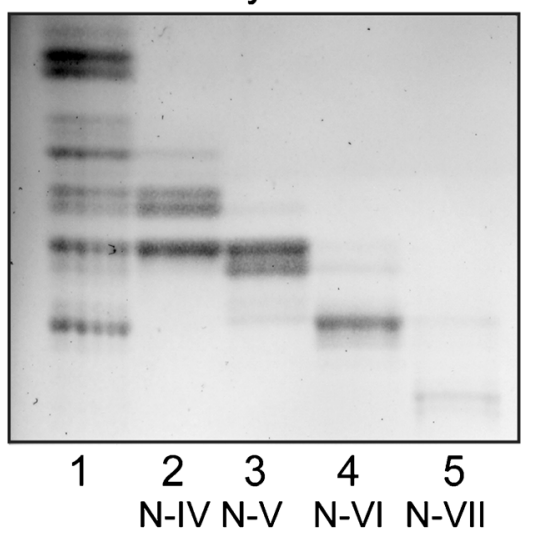

b E. cristagalli lectin

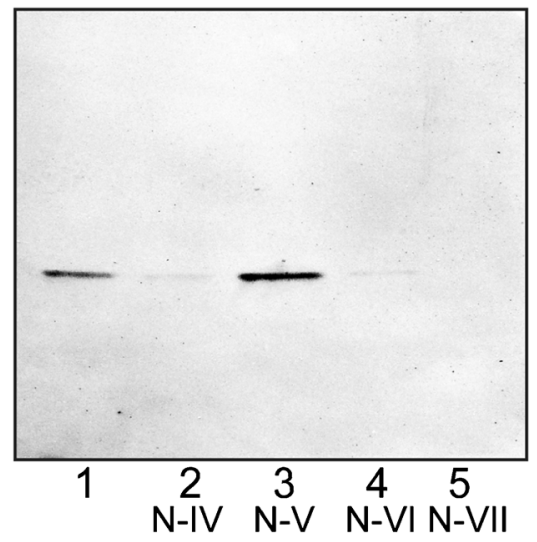

c Anti-H type 1

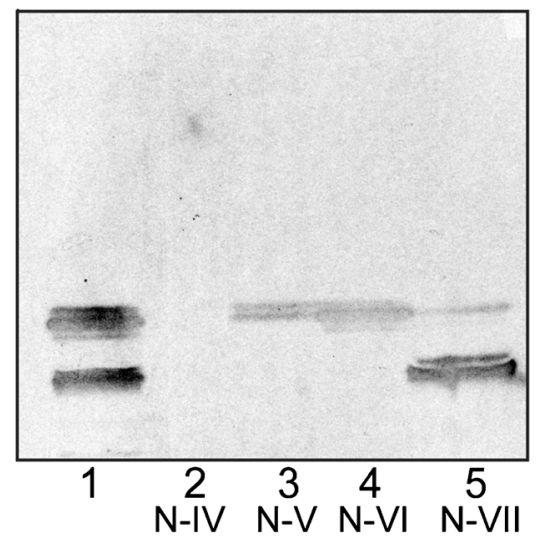

d G. simplicifolia IB4 lectin
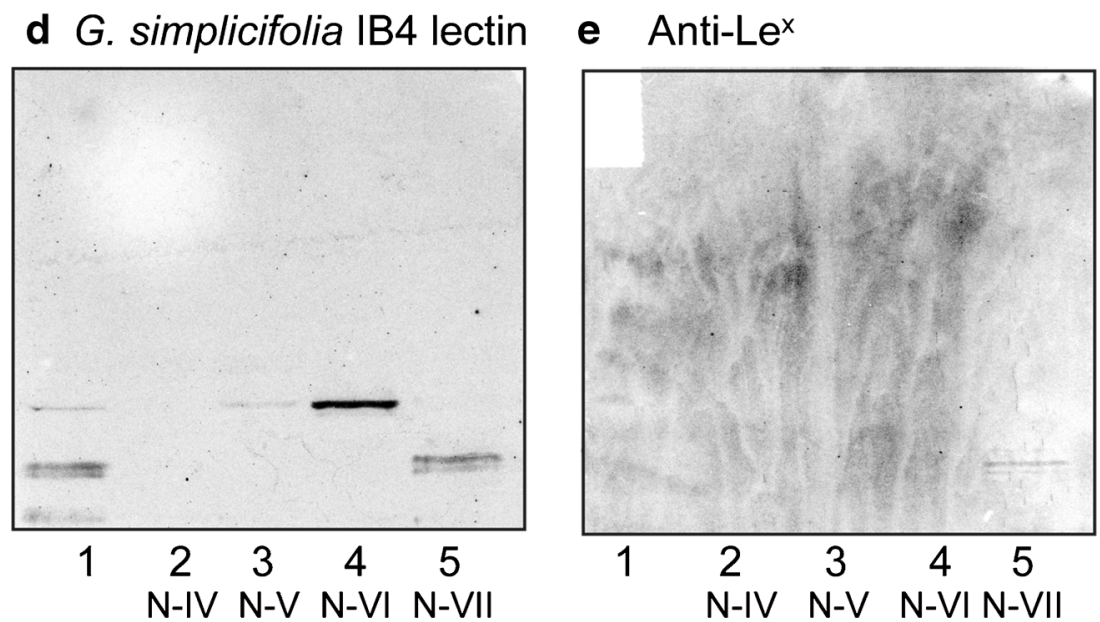

binding of the anti Lewis ${ }^{\mathrm{x}}$ antibodies to fraction N-VII (heptaglycosylceramide region) was also observed (Fig. 4e).

\section{Mass spectrometry}

Fractions N-I and N-II The base chromatogram from ESI/ MS of fraction N-I had a number of molecular ions corresponding to monoglycosylceramides, while the molecular ions in the base chromatogram of fraction N-II indicated mono- and diglycosylceramides (data not shown). The major monoglycosylceramide ions were found at $\mathrm{m} / \mathrm{z} 816$ and $\mathrm{m} / \mathrm{z}$ 844 , corresponding to the species with t18:0-h22:0 and t18:0$\mathrm{h} 24: 0$, respectively. The diglycosylceramide ions were found at $\mathrm{m} / \mathrm{z} 860, \mathrm{~m} / \mathrm{z} 876, \mathrm{~m} / \mathrm{z} 894, \mathrm{~m} / \mathrm{z} 978$, and $\mathrm{m} / \mathrm{z} 1006$, corresponding to the species with d18:1-16:0, d18:1-h16:0, t18:0h16:0, t18:0-h22:0 and t18:0-h24:0, respectively. 
Fractions N-III to N-VII Fractions N-III to N-VII were hydrolyzed with Rhodococcus endoglycoceramidase II, and the oligosaccharides thereby obtained were analyzed by LC-ESI/ MS using graphitized carbon columns. This gives a resolution of isomeric oligosaccharides, and the carbohydrate sequence may be deduced from series of C-type fragment ions obtained by $\mathrm{MS}^{2}$ [6]. $\mathrm{MS}^{2}$ spectra of oligosaccharides with a Hex or HexNAc substituted at C-4 have diagnostic cross-ring ${ }^{0,2} \mathrm{~A}$ type fragment ions, which allow differentiation of linkage positions.

The base peak chromatogram from LC-ESI/MS of the oligosaccharides obtained from the total non-acid glycosphingolipid fraction from moose I small intestine is shown in Fig. 5a. Molecular ions corresponding to oligosaccharides ranging from trisaccharides (detected as $\left[\mathrm{M}-\mathrm{H}^{+}\right]^{-}$ ions at $m / z 503$ ) to heptasaccharides (detected as $\left[\mathrm{M}-\mathrm{H}^{+}\right]^{-}$ions at $\mathrm{m} / \mathrm{z} 1217$ and 1233) were found. In addition, a number of minor oligosaccharides were detected in the mass chromatograms from LC-ESI/MS of fractions N-III to N-VII (Fig. 5bf).

Fractions N-III, N-IV and N-V $\mathrm{MS}^{2}$ of the molecular ions in the base peak chromatograms of fractions N-III and N-IV (Fig. $5 b$ and $c$ ) allowed a tentative identification of a number of glycosphingolipid derived oligosaccharides. The $\mathrm{MS}^{2}$ data are summarized in Table 2. Thus, a globo trisaccharide (Gal $\alpha 4 \mathrm{Gal} \beta 4 \mathrm{Glc})$, a globo tetrasaccharide (GalNAc $\beta 3 \mathrm{Gal} \alpha 4 \mathrm{Gal} \beta 4 \mathrm{Glc}$ ), and an $\mathrm{H}$ trisaccharide (Fuc $\alpha 2 \mathrm{Gal} \beta 4 \mathrm{Glc}$ ) were present in fraction N-III, while fraction N-IV had a globo trisaccharide, a globo tetrasaccharide, a lacto trisaccharide ( $\mathrm{GlcNAc} \beta 3 \mathrm{Gal} \beta 4 \mathrm{Glc})$, a lacto tetrasaccharide $(\mathrm{Gal} \beta 3 \mathrm{GlcNAc} \beta 3 \mathrm{Gal} \beta 4 \mathrm{Glc})$, and a neolacto tetrasaccharide (Gal $\beta 4 \mathrm{GlcNAc} \beta 3 \mathrm{Gal} \beta 4 \mathrm{Glc})$.

The base peak chromatogram of the oligosaccharides derived from fraction N-V (Fig. 5d) showed that this fraction was a mixture of compounds found in fraction N-IV and fraction N-VI. The only unique feature was the ion at $\mathrm{m} / \mathrm{z} 503$ eluting at $24.4 \mathrm{~min}$. Here $\mathrm{MS}^{2}$ gave a $\mathrm{B}_{1}$ ion at $\mathrm{m} / z$ 161, and a C-type ion series $\left(\mathrm{C}_{1}\right.$ at $\mathrm{m} / \mathrm{z} 179$ and $\mathrm{C}_{2}$ at $\left.\mathrm{m} / \mathrm{z} 341\right)$, demonstrating a Hex-Hex-Hex sequence. There was no ${ }^{0,2} \mathrm{~A}_{2}$ fragment ion at $m / z$ 281, suggesting that the internal Hex was substituted at C-3. Taken together, these spectral features indicated the presence of a isoglobotrisaccharide (Gal $\alpha 3 \mathrm{Gal} \beta 4 \mathrm{Glc})$.

Fraction N-VI The base peak chromatogram of fraction $\mathrm{N}-\mathrm{VI}$ (Fig. 5e) had two molecular ions at $\mathrm{m} / \mathrm{z} 852$, one major eluting at 27.6-28.6 min and one minor eluting at

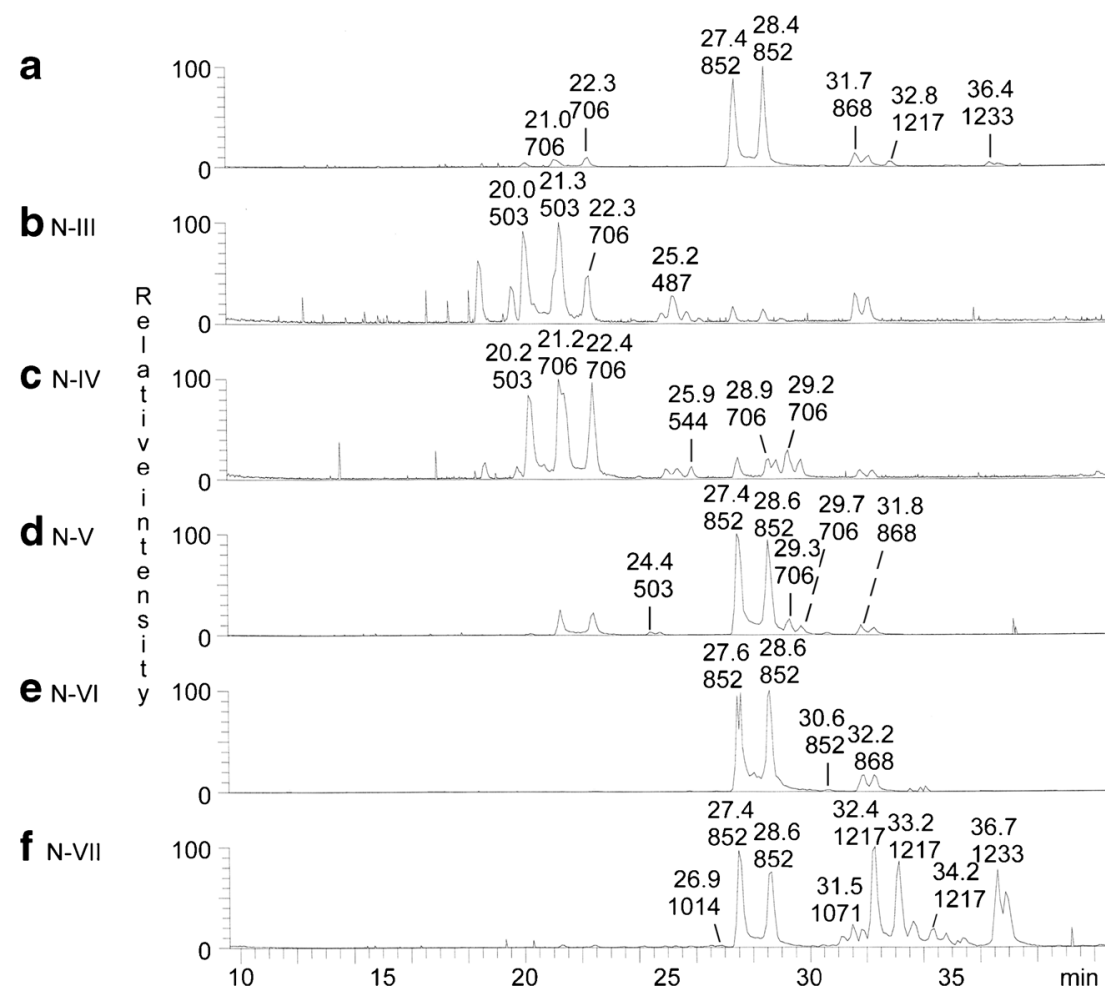

Fig. 5 LC-ESI/MS of the oligosaccharides obtained by digestion of nonacid glycosphingolipids from moose I small intestine with Rhodococcus endoglycoceramidase II. a Base peak chromatogram from LC-ESI/MS of the oligosaccharides obtained by digestion of the total non-acid glycosphingolipid fraction from moose I small intestine. b Base peak chromatogram from LC-ESI/MS of the oligosaccharides derived from

fraction N-III. c Base peak chromatogram from LC-ESI/MS of the oligosaccharides derived from fraction N-IV. d Base peak chromatogram from LC-ESI/MS of the oligosaccharides derived from fraction N-V. e Base peak chromatogram from LC-ESI/MS of the oligosaccharides derived from fraction N-VI. f Base peak chromatogram from LC-ESI/MS of the oligosaccharides derived from fraction N-VII 
Table 2 LC-ESI/MS of the oligosaccharides obtained by digestion of the non-acid fractions N-III and N-IV from moose I small intestine with Rhodococcus endoglycoceramidase II. Summary of $\mathrm{MS}^{2}$ data

\begin{tabular}{lllllll}
\hline Fraction & $\begin{array}{l}{\left[\mathrm{M}-\mathrm{H}^{+}\right]^{-}} \\
\text {ion }\end{array}$ & $\mathrm{RT}(\mathrm{min})$ & $\mathrm{C}$ ions $(m / z)$ & $\begin{array}{l}{ }^{0,2} \mathrm{~A} \text { ions } \\
(m / z)^{\mathrm{a}}\end{array}$ & Carbohydrate sequence & Tentative oligosaccharide \\
\hline $\mathrm{N}$-III & $m / z 503$ & $20.0 / 21.3$ & $\mathrm{C}_{1} 179 ; \mathrm{C}_{2} 341$ & ${ }^{0,2} \mathrm{~A}_{2} 281$ & Hex-4Hex-4Hex & Gal $\alpha 4 \mathrm{Gal} \beta 4 \mathrm{Glc}$ \\
& $m / z 706$ & 22.3 & $\mathrm{C}_{1} 220 ; \mathrm{C}_{2} 382 ; \mathrm{C}_{3} 544$ & ${ }^{0,2} \mathrm{~A}_{3} 484$ & HexNAc-Hex-4Hex-4Hex & GalNAc $\beta 3 \mathrm{Gal} \alpha 4 \mathrm{Gal} \beta 4 \mathrm{Glc}$ \\
& $m / z 487$ & 25.2 & $\mathrm{C}_{1} 163 ; \mathrm{C}_{2} 325$ & - & Fuc-Hex-4Hex & Fuc $\alpha 2 \mathrm{Gal} \beta 4 \mathrm{Glc}$ \\
$\mathrm{N}-\mathrm{IV}$ & $m / z 503$ & 20.2 & $\mathrm{C}_{1} 179 ; \mathrm{C}_{2} 341$ & ${ }^{0,2} \mathrm{~A}_{2} 281$ & Hex-4Hex-4Hex & Gal $\alpha 4 \mathrm{Gal} \beta 4 \mathrm{Glc}$ \\
& $m / z 706$ & $21.2 / 22.4$ & $\mathrm{C}_{1} 220 ; \mathrm{C}_{2} 382 ; \mathrm{C}_{3} 544$ & ${ }^{0,2} \mathrm{~A}_{3} 484$ & HexNAc-Hex-4Hex-4Hex & GalNAc $\beta 3 \mathrm{Gal} \alpha 4 \mathrm{Gal} \beta 4 \mathrm{Glc}$ \\
& $m / z 544$ & 25.9 & $\mathrm{C}_{1}$ at $m / z 220, \mathrm{C}_{2}$ at $m / z 382$ & - & HexNAc-Hex-4Hex & GlcNAc $\beta 3 \mathrm{Gal} \beta 4 \mathrm{Glc}$ \\
& $m / z 706$ & 28.9 & $\mathrm{C}_{2} 382 ; \mathrm{C}_{3}$ at $m / z 544$ & - & Hex-HexNAc-Hex-4Hex & Gal $\beta 3 \mathrm{GlcNAc} \beta 3 \mathrm{Gal} \beta 4 \mathrm{Glc}$ \\
& $m / z 706$ & 29.2 & $\mathrm{C}_{2} 382 ; \mathrm{C}_{3}$ at $m / z 544$ & ${ }^{0,2} \mathrm{~A}_{2} 281$ & Hex-4HexNAc-Hex-4Hex & Gal $\beta 4 \mathrm{GlcNAc} \beta 3 \mathrm{Gal} \beta 4 \mathrm{Glc}$ \\
\hline
\end{tabular}

${ }^{\mathrm{a}}$ The ${ }^{0,2} \mathrm{~A}$ ions derived from the lactose unit at the reducing end are not given

$30.6 \mathrm{~min} . \mathrm{MS}^{2}$ gave in both cases a series of $\mathrm{C}$ type fragment ions $\left(\mathrm{C}_{2}\right.$ at $m / z 325, \mathrm{C}_{3}$ at $m / z 528$ and $\mathrm{C}_{4}$ at $\mathrm{m} / \mathrm{z}$ 690) identifying a Fuc-Hex-HexNAc-Hex-Hex oligosaccharide (Fig. 6a and b). The $\mathrm{MS}^{2}$ spectrum of the minor ion eluting at $30.6 \mathrm{~min}$ (Fig. 6b) also had a prominent ${ }^{0,2} \mathrm{~A}_{3}-\mathrm{H}_{2} \mathrm{O}$ fragment ion at $m / z 409$ and a ${ }^{0,2} \mathrm{~A}_{3}$ fragment ion at $m / z 427$, characteristic for 4-substituted HexNAc, i.e., type 2 carbohydrate chains. The features of the two spectra were very similar to those of reference $\mathrm{H}$ type 1 and $\mathrm{H}$ type 2 pentasaccharides, respectively [6], which allowed identification of one Fuc $\alpha 2 \mathrm{Gal} \beta 3 \mathrm{GlcNAc} \beta 3 \mathrm{Gal} \beta 4 \mathrm{Glc}$ saccharide (major ion) and one Fuc $\alpha 2 \mathrm{Gal} \beta 4 \mathrm{GlcNAc} \beta 3 \mathrm{Gal} \beta$ 4Glc saccharide (minor ion).

A molecular ion at $m / z 868$ was also present in the base peak chromatogram of fraction N-VI (Fig. 5e). Here, $\mathrm{MS}^{2}$ (Fig. 6c) gave a tentative identification of a Gal $\alpha 3$ Gal $\beta 4$ GlcNAc-terminated pentasaccharide (Gal $\alpha 3 \mathrm{Gal} \beta 4 \mathrm{GlcNAc} \beta 3 \mathrm{Gal} \beta 4 \mathrm{Glc})$. This was concluded from the C-type fragment ion series $\left(\mathrm{C}_{2}\right.$ at $m / z 341, \mathrm{C}_{3}$ at $m / z$ 544, and $\mathrm{C}_{4}$ at $m / z$ 706) identifying a Hex-HexHexNAc-Hex-Hex sequence, and the ${ }^{0,2} \mathrm{~A}_{2}-\mathrm{H}_{2} \mathrm{O}$ fragment ion at $m / z 425$, and the ${ }^{0,2} \mathrm{~A}_{3}$ fragment ion at $\mathrm{m} / \mathrm{z} 443$, demonstrating a substitution of the HexNAc at C-4, i.e., a type 2 chain.

Fraction N-VII The base peak chromatogram of the oligosaccharides obtained from fraction N-VII showed that this fraction was a complex mixture (Fig. 5f). Also here an $\mathrm{H}$ type 1 pentasaccharide was characterized by $\mathrm{MS}^{2}$ of the ion at $m / z 852$.

$\mathrm{MS}^{2}$ of oligosaccharides with terminal Le ${ }^{\mathrm{x}}(\mathrm{Gal} \beta 4$ (Fuc $\alpha 3)$ GlcNAc-) sequence gives a fragment ion at $m / z$ 364. This fragment ion is obtained by double glycosidic cleavage of the 3-linked branch at $\mathrm{C}_{3}$ and $\mathrm{Z}_{3 \beta}$, and is characteristic for an internal 4-linked GlcNAc substituted with a Fuc at 3position [14]. The base peak chromatogram of fraction NVII (Fig. 5f) had a minor ion at $m / z 1014$, corresponding to an oligosaccharide with one Fuc, one HexNAc and four Hex.
$\mathrm{MS}^{2}$ (Fig. 6d) gave an ion at $m / z 526$, which along with the $\mathrm{C}_{2}$ ion at $\mathrm{m} / \mathrm{z} 341$ denoting a terminal Hex-Hex sequence, indicated a Hex-Gal $\beta 4$ (Fuc $\alpha 3$ )GlcNAc terminal. Furthermore, there was a $\mathrm{C}_{4}$ ion at $\mathrm{m} / \mathrm{z} 852$. Taken together, these spectral features indicated the presence of a Gal $\alpha 3-\mathrm{Le}^{\mathrm{x}}$ hexasaccharide (Gal $\alpha 3$ Gal $\beta 4$ (Fuc $\alpha 3$ )GlcNAc $\beta 3$ Gal $\beta 4$ Glc), previously characterized among porcine kidney glycosphingolipids [15].

The base chromatogram of fraction N-VII (Fig. 5f) also had a molecular ion at $m / z 1071$, and $\mathrm{MS}^{2}$ of this ion (Fig. 6e) gave a preliminary identification of a lacto-neolacto hexasaccharide (Gal $\beta 3$ GlcNAc $\beta 3$ Gal $\beta 4$ GlcNAc $\beta 3$ Gal $\beta 4$ Glc). This was concluded from the C-type fragment ions $\left(\mathrm{C}_{2}\right.$ at $\mathrm{m} / z$ 382, $\mathrm{C}_{3}$ at $m / z 544, \mathrm{C}_{4}$ at $m / z 747$, and $\mathrm{C}_{5}$ at $m / z 909$ ) identifying a Hex-HexNAc-Hex-HexNAc-Hex-Hex sequence, along with the prominent ${ }^{0,2} \mathrm{~A}_{4}-\mathrm{H}_{2} \mathrm{O}$ fragment ion at $m / z 628$, and the ${ }^{0}$, ${ }^{2} \mathrm{~A}_{4}$ fragment ion at $m / z 646$, demonstrating 4-substitution of the HexNAc at the reducing end. $\mathrm{MS}^{3}$ of $m / z 646$ gave no ${ }^{0}$, ${ }^{2} \mathrm{~A}_{2}$ fragment ion at $m / z 281$, indicating that the penultimate HexNAc was substituted at C-3 (data not shown). The absence of binding of the Gal 34 GlcNAc-recognizing lectin from E. cristagalli to fraction N-VII (Fig. 4b, lane 5) is consistent with this suggestion.

The two molecular ions at $\mathrm{m} / \mathrm{z} 1217$ in the base peak chromatogram of fraction N-VII (Fig. 5f) eluted at 32.4-33.2 min and $34.2 \mathrm{~min}$, respectively. Here the $\mathrm{MS}^{2}$ spectra obtained were very similar (Fig. $6 \mathrm{f}$ and $\mathrm{g}$ ), both having $\mathrm{C}$-type ion series $\left(\mathrm{C}_{3}\right.$ at $\mathrm{m} / z$ 528, $\mathrm{C}_{4}$ at $\mathrm{m} / z$ 690, $\mathrm{C}_{5}$ at $\mathrm{m} / z$ 893, and $\mathrm{C}_{6}$ at $m / z 1055) . \mathrm{MS}^{3}$ of $m / z 690$ gave in both cases a prominent $\mathrm{C}_{2}$ ion at $\mathrm{m} / \mathrm{z} 325$, demonstrating a terminal Fuc-Hex sequence (data not shown), and taken together this demonstrated a heptasaccharide with Fuc-Hex-HexNAc-Hex-HexNAc-HexHex sequence. In the $\mathrm{MS}^{2}$ spectrum of the ion eluting at $34.2 \mathrm{~min}$ there was also a ${ }^{0,2} \mathrm{~A}_{5}-\mathrm{H}_{2} \mathrm{O}$ ion at $\mathrm{m} / \mathrm{z} 774$, and a ${ }^{0}$, ${ }^{2} \mathrm{~A}_{5}$ ion at $m / z 792$, demonstrating that the reducing end HexNAc was substituted at C-4 (Fig. 6g). Furthermore, upon closer inspection of the low molecular range a ${ }^{0,2} \mathrm{~A}_{3}-\mathrm{H}_{2} \mathrm{O}$ ion at $\mathrm{m} / \mathrm{z} 409$, and a ${ }^{0,2} \mathrm{~A}_{5}$ ion at $\mathrm{m} / \mathrm{z} 427$, were found, 
Fig. 6 LC-ESI/MS of the oligosaccharides obtained by digestion of non-acid glycosphingolipids (fraction NVI and N-VII) from moose I small intestine with Rhodococcus endoglycoceramidase II. a $\mathrm{MS}^{2}$ of the ion at $\mathrm{m} / \mathrm{z} 852$ (retention time $27.5 \mathrm{~min}$ ) from LC-ESI/MS of fraction N-VI. b $\mathrm{MS}^{2}$ of the ion at $\mathrm{m} / \mathrm{z} 852$ (retention time $30.7 \mathrm{~min}$ ) from LC-ESI/MS of fraction N-VI. $\mathbf{c} \mathrm{MS}^{2}$ of the ion at $\mathrm{m} / \mathrm{z} 868$ (retention time $32.1 \mathrm{~min}$ ) from LC-ESI/MS of fraction NVI. d $\mathrm{MS}^{2}$ of the ion at $\mathrm{m} / \mathrm{z} 1014$ (retention time $26.6 \mathrm{~min}$ ) from LC-ESI/MS of fraction N-VII. e $\mathrm{MS}^{2}$ of the ion at $\mathrm{m} / z 1071$ (retention time $31.4 \mathrm{~min}$ ) from LC-ESI/MS of fraction N-VII. $\mathrm{f} \mathrm{MS}^{2}$ of the ion at $\mathrm{m} / \mathrm{z} 1217$ (retention time $32.8 \mathrm{~min}$ ) from LC-ESI/MS of fraction N-VII. g MS $^{2}$ of the ion at $m / z 1217$ (retention time $34.2 \mathrm{~min}$ ) from LC-ESI/MS of fraction N-VII. h $\mathrm{MS}^{2}$ of the ion at $\mathrm{m} / \mathrm{z} 1233$ (retention time $37.7 \mathrm{~min}$ ) from LC-ESI/MS of fraction N-VII. i Interpretation formulas showing the deduced carbohydrate sequences

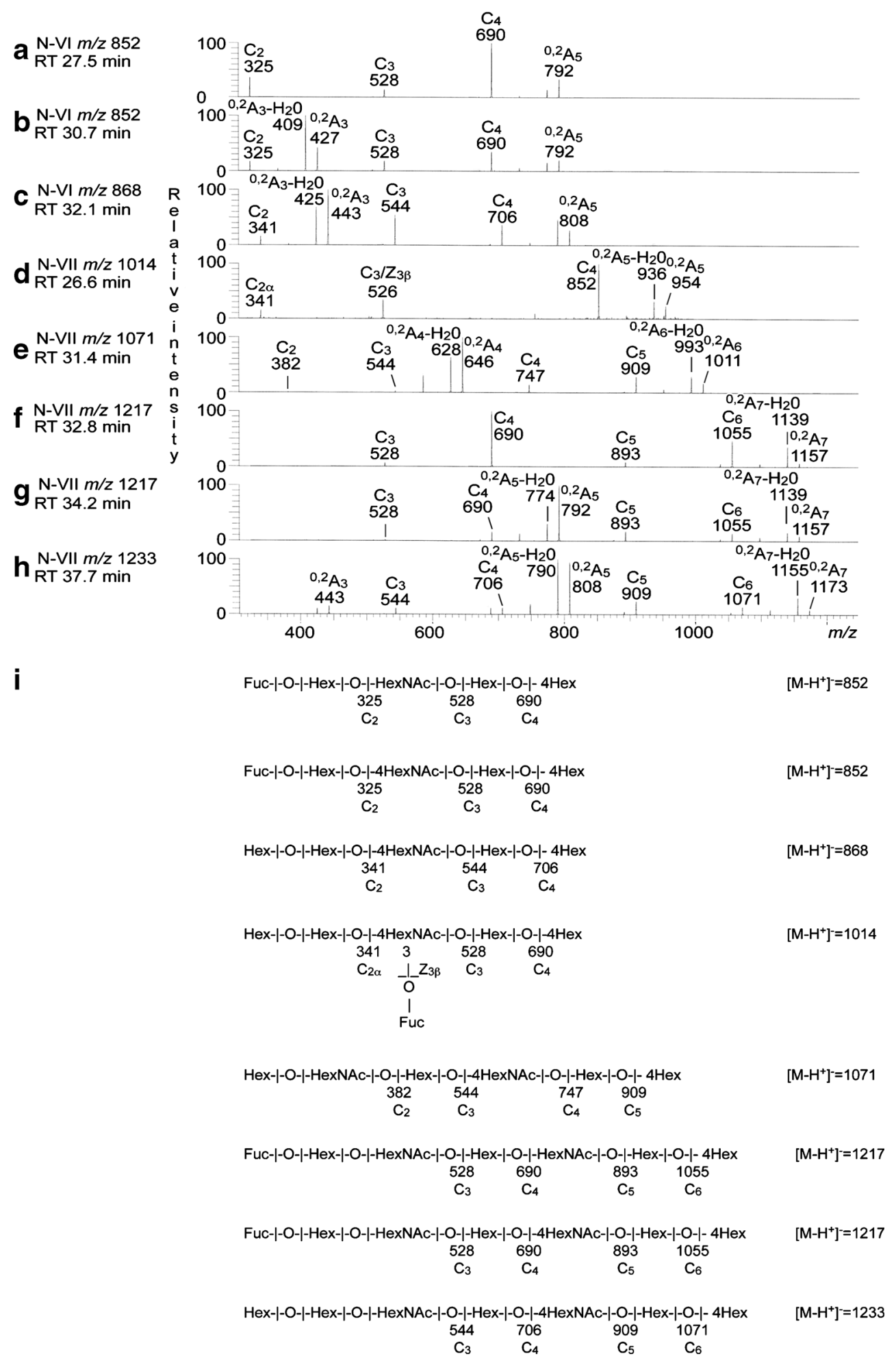

demonstrating 4-substitution also of the first HexNAc (data not shown). Taken together these spectral features allowed tentative identification of one $\mathrm{H}$ type 1 heptasaccharide (Fuc $\alpha 2$ Gal $\beta 3$ GlcNAc $\beta 3$ Gal $\beta 3$ GlcNAc $\beta$ $3 \mathrm{Gal} \beta 4 \mathrm{Glc})$ and one $\mathrm{H}$ type 2 heptasaccharide (Fuc $\alpha 2 \mathrm{Gal} \beta 4 \mathrm{GlcNAc} \beta 3 \mathrm{Gal} \beta 4 \mathrm{GlcNAc} \beta 3 \mathrm{Gal} \beta 4 \mathrm{Glc}$ ).
Finally, a heptasaccharide with type 2 core chain and HexHex-HexNAc-Hex-HexNAc-Hex-Hex sequence was suggested by $\mathrm{MS}^{2}$ of the ion at $\mathrm{m} / \mathrm{z} 1233$ of fraction N-VII (Fig. 6h). This was concluded from the C-type ion series $\left(\mathrm{C}_{3}\right.$ at $m / z 544, \mathrm{C}_{4}$ at $\mathrm{m} / \mathrm{z} 706, \mathrm{C}_{5}$ at $\mathrm{m} / \mathrm{z} 909$, and $\mathrm{C}_{6}$ at $\mathrm{m} / \mathrm{z} 1071$ ), along with the ${ }^{0,2} \mathrm{~A}_{3}$ fragment ion at $m / z 443$, and the ${ }^{0,2} \mathrm{~A}_{5^{-}}$ 
$\mathrm{H}_{2} \mathrm{O}$ fragment ion at $m / z 790$, and the ${ }^{0,2} \mathrm{~A}_{5}$ fragment ion at $\mathrm{m} / \mathrm{z} 808$, demonstrating that the two HexNAcs were substituted at C-4. Upon $\mathrm{MS}^{3}$ of $m / z 706$ a prominent $\mathrm{C}_{2}$ ion at $\mathrm{m} / \mathrm{z} 341$ was observed, demonstrating a terminal Hex-Hex sequence (data not shown). Thus, these spectral features suggested a Gal $\alpha 3$ Gal $\beta 4$ GlcNAc-terminated heptasaccharide (Gal $\alpha 3 \mathrm{Gal} \beta 4 \mathrm{GlcNAc} \beta 3 \mathrm{Gal} \beta 4 \mathrm{GlcNAc} \beta 3 \mathrm{Gal} \beta 4 \mathrm{Glc}$ ).

In summary, chromatogram binding assays and mass spectrometry allowed identification of a number of non-acid glycosphingolipids of moose I small intestine, i.e., monoand dihexosylceramides, globotriaosylceramide, lactotriaosylceramide, isoglobotriaosylceramide, blood group $\mathrm{H}$ triaosylceramide, globotetraosylceramide, lactotetraosylceramide, neolactotetraosylceramide, blood group $\mathrm{H}$ type 1 pentaosylceramide, blood group $\mathrm{H}$ type 2 pentaosylceramide, Galili pentaosylceramide, Gal $\alpha 3-\mathrm{Le}^{\mathrm{x}}$ hexaosylceramide, lacto-neolactohexaosylceramide, $\mathrm{H}$ type 1 heptaosylceramide, $\mathrm{H}$ type 2 heptaosylceramide and Galili heptaosylceramide, were identified.

\section{Characterization of the non-acid glycosphingolipids of moose II small intestine}

In the case of moose II small intestine we focused on characterization of the major compounds recognized by antibodies directed against the blood group A determinant. To this end the total non-acid fraction was separated into ten fractions, and by binding of anti-A antibodies, and BabA expressing Helicobacter pylori strain J99 (Fig. 7b and c, lanes 2-4), three fractions were selected for structural characterization. In the first fraction (denoted fraction N2-IV) the anti-A antibodies bound in the tetraosylceramide region (Fig. 7b). The second fraction (denoted fraction N2-VII) had anti-A binding in the hexa- and octaosylceramide regions, while in the third fraction (denoted fraction N2-IX) the antibodies bound in octaosylceramide region and to a slow-migrating compound. The compounds migrating in the hexa- and octaosylceramide regions, and the slow-migrating compound, were also recognized by the $H$. pylori strain $\mathrm{J} 99$ with generalist BabA [16] (Fig. 7c).

Fraction N2-IV The base peak chromatogram from LC-ESI/ MS of the oligosaccharides of fraction N2-IV (Fig. 7d; Supplementary Figure 1A) had a number of molecular ions at $\mathrm{m} / \mathrm{z}$ 503, $\mathrm{m} / \mathrm{z} 706$ and $\mathrm{m} / \mathrm{z}$ 487. By MS ${ }^{2}$ of these ions allowed identification of globo trisaccharide, globo tetrasaccharide, an $\mathrm{H}$ trisaccharide, and a neolacto tetrasaccharide, as in the small intestine of moose I above.

In addition there was a minor molecular ion at $m / z 690$, and the $\mathrm{MS}^{2}$ spectrum obtained of this ion (Supplementary Figure 1C) demonstrated a blood group A tetrasaccharide (GalNAc $\alpha 3$ (Fuc $\alpha 2$ )Gal $\beta 4$ Glc). This was concluded from the C-type fragment ions $\left(\mathrm{C}_{1 \alpha}\right.$ at $\mathrm{m} / \mathrm{z} 220$ and $\mathrm{C}_{2}$ at $\left.\mathrm{m} / \mathrm{z} 528\right)$ identifying a HexNAc-(Fuc-)Hex-Hex sequence, along with the binding of anti-A antibodies in the tetraglycosylceramide region of this fraction (Fig. 7b, lane 2). The ${ }^{0,2} \mathrm{~A}_{3}$ fragment ion at $m / z 630$, and the ${ }^{0,2} \mathrm{~A}_{2}-\mathrm{H}_{2} \mathrm{O}$ fragment ion at $m / z 612$, were derived from cross-ring cleavage of the 4-substituted Glc of the lactose unit at the reducing end.

Fraction N2-VII Molecular ions at $\mathrm{m} / \mathrm{z} 1055, \mathrm{~m} / \mathrm{z}$ 1420, $\mathrm{m} / \mathrm{z}$ 868 and $\mathrm{m} / z 1233$ were present in the base peak chromatogram of fraction N2-VII (Fig. 7e; Supplementary Figure 1B). As above, a Galili penta- and heptasaccharide were identified by $\mathrm{MS}^{2}$ of the ions at $\mathrm{m} / \mathrm{z} 868$ and $\mathrm{m} / \mathrm{z} 1233$, respectively.

$\mathrm{MS}^{2}$ of the ion at $\mathrm{m} / z 1055$ (Supplementary Figure 1D) gave a series of C-type fragment ions $\left(\mathrm{C}_{2}\right.$ at $\mathrm{m} / \mathrm{z} 528, \mathrm{C}_{3}$ at $m / z$ 731, and $\mathrm{C}_{4}$ at $m / z$ 893) indicating a HexNAc-(Fuc)HexHexNAc-Hex-Hex sequence. There was no ${ }^{0,2} \mathrm{~A}_{3}$ fragment ion at $m / z 630$, suggesting that the internal HexNAc was substituted at $\mathrm{C} 3$, i.e., a type 1 core chain. This $\mathrm{MS}^{2}$ spectrum was very similar to the $\mathrm{MS}^{2}$ spectrum of reference blood group A type 1 hexasaccharide [6]. Thus, a hexasaccharide with HexNAc-(Fuc)Hex-HexNAc-Hex-Hex sequence and an internal HexNAc substituted at C-3 was indicated, most likely a blood group A type 1 hexasaccharide (GalNAc $\alpha 3$ (Fuc $\alpha 2)$ $\mathrm{Gal} \beta 3 \mathrm{GlcNAc} \beta 3 \mathrm{Gal} \beta 4 \mathrm{Glc})$.

The same series of C-type fragment ions $\left(\mathrm{C}_{2}\right.$ at $\mathrm{m} / z 528, \mathrm{C}_{3}$ at $m / z 731$, and $\mathrm{C}_{4}$ at $m / z$ 893) was obtained by $\mathrm{MS}^{2}$ of the ion at $m / z 1420$ (Supplementary Figure 1E). In addition, there was a $C_{5}$ ion at $m / z 1096$ and a $C_{6}$ ion at $m / z$ 1258. Taken together this demonstrated a HexNAc-(Fuc)Hex-HexNAc-HexHexNAc-Hex-Hex sequence. Type 1 core chain was indicated by the absence of ${ }^{0,2} \mathrm{~A}_{3}$ and ${ }^{0,2} \mathrm{~A}_{5}$ fragment ions. Thus, a blood group A type 1 octasaccharide (GalNAc $\alpha 3(\mathrm{Fuc} \alpha 2)$ Gal $\beta 3 \mathrm{GlcNAc} \beta 3 \mathrm{Gal} \beta 3 \mathrm{GlcNAc} \beta 3 \mathrm{Gal} \beta 4 \mathrm{Glc})$ was tentatively identified.

Fraction N2-IX LC-ESI/MS of the oligosaccharides obtained by hydrolysis of fraction N2-IX (Fig. 7f) gave a major $\left[\mathrm{M}-2 \mathrm{H}^{+}\right]^{2-}$ ion at $\mathrm{m} / \mathrm{z} 1067$, corresponding to a $\left[\mathrm{M}-\mathrm{H}^{+}\right]^{-}$ion at $m / z 2134$, demonstrating a dodecasaccharide with two Fuc, five HexNAc and five Hex. MS ${ }^{2}$ of $m / z 1067$ gave a weak lower mass region (Fig. $7 \mathrm{~g}$ ). However, there was a $\mathrm{C}_{2}$ ion at $\mathrm{m} / \mathrm{z} 528$ and $\mathrm{a}_{3}$ ion at $\mathrm{m} / \mathrm{z} 731$, indicating a terminal HexNAc-(Fuc-)Hex-HexNAc sequence. In addition, there were intense C type ions at $\mathrm{m} / \mathrm{z} 1607, \mathrm{~m} / \mathrm{z} 1810$, and $\mathrm{m} / \mathrm{z}$ 1972. The $\mathrm{MS}^{3}$ spectrum of the ion at $\mathrm{m} / z 1607$ also had a $\mathrm{C}_{2}$ ion at $\mathrm{m} / \mathrm{z} 528$ and a $\mathrm{C}_{3}$ ion at $\mathrm{m} / \mathrm{z} 731$ (Fig. 7h). This spectrum was dominated by a ${ }^{0,2} \mathrm{~A}_{3}$ ion at $\mathrm{m} / \mathrm{z} 630$, and a ${ }^{0,2} \mathrm{~A}_{3}-\mathrm{H}_{2} \mathrm{O}$ ion at $\mathrm{m} / \mathrm{z} 612$, which together with the $\mathrm{C}_{2}$ ion at $\mathrm{m} / \mathrm{z} 528$ and a $\mathrm{C}_{3}$ ion at $\mathrm{m} / \mathrm{z} 731$, identified indicating a terminal HexNAc-(Fuc-)Hex-HexNAc sequence sequence with 4-substitution of the internal HexNAc, i.e., a type 2 core chain. Finally, a $\mathrm{C}_{1}$ ion at $\mathrm{m} / \mathrm{z} 220$, confirming a terminal HexNAc, was obtained by $\mathrm{MS}^{4}$ of 

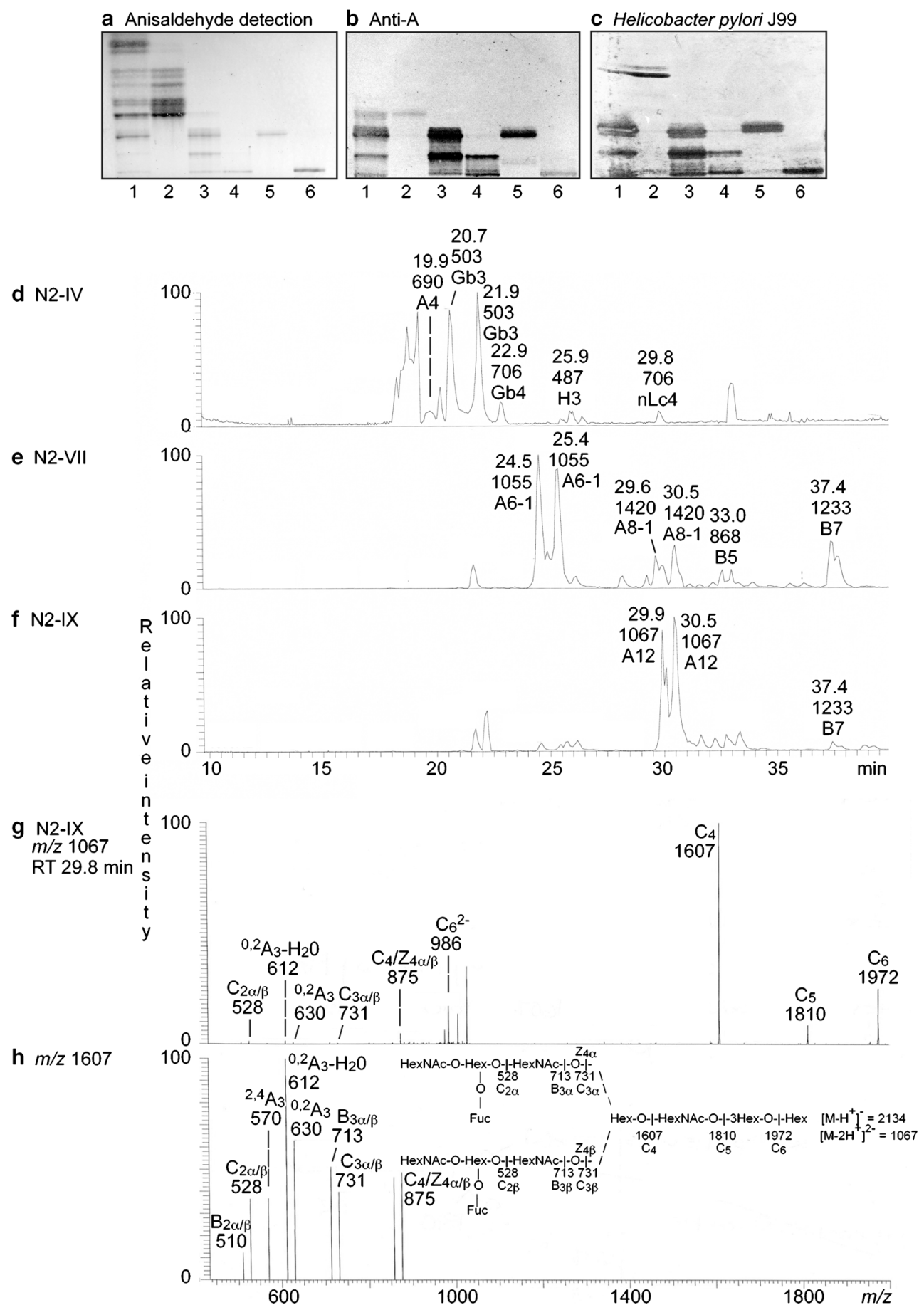

$m / z 612$ (data not shown). Thus, the $\mathrm{MS}^{2}, \mathrm{MS}^{3}$ and $\mathrm{MS}^{4}$ spectral features suggested that fraction N2-IX contained a branched dodecasaccharide with HexNAc-(Fuc-)HexHexNAc-(HexNAc-(Fuc-)Hex-HexNAc-)Hex-HexNAcHex-Hex sequence, and type 2 core on at least one of the HexNAc-(Fuc-)Hex-HexNAc branches. However, the binding of $H$. pylori strain $\mathrm{J} 99$ with generalist BabA to the slow-migrating dodecaosylceramide in fraction
N-IX (Fig. 7c) indicated the presence of a type 1 core chain, since this variant of $H$. pylori BabA adhesin does not bind to blood group A or B determinants on type 2 core $[12,16]$. Thus, fraction N-IX either contained two dodecaosylceramides with blood group A determinants on type 1 and type 2 core branches, or one dodecaosylceramide with blood group A determinants on one type 1 and one type 2 core branch. 
Fig. 7 Characterization of the non-acid glycosphingolipids from moose II small intestine. Thin-layer chromatogram after detection with anisaldehyde (a), and autoradiograms obtained by the monoclonal antiA antibody HE193 (b), and BabA expressing Helicobacter pylori strain J99 (c). The glycosphingolipids were separated on aluminum-backed silica gel plates, using chloroform/methanol/water 60:35:8 (by volume) as solvent system, and the binding assays were performed as described under " Materials and methods". Autoradiography was for $12 \mathrm{~h}$. The lanes were: Lane 1, total non-acid glycosphingolipids of moose II small intestine, $40 \mu \mathrm{g}$; Lane 2, fraction N2-IV from moose II small intestine, $4 \mu \mathrm{g}$; Lane 3, fraction N2-VII from moose II small intestine, $4 \mu \mathrm{g}$; Lane 4, fraction N2-IX from moose II small intestine, $4 \mu \mathrm{g}$; Lane 5, reference A type 1 hexaosylceramide (GalNAc $\alpha 3$ (Fuc $\alpha 2$ ) Gal $\beta 3$ GlcNAc $\beta 3$ Gal $\beta 4$ Glc $\beta 1$ Cer), $4 \mu \mathrm{g}$; Lane 6 , reference A type 1 dodecaosylceramide (GalNAc $\alpha 3$ (Fuc $\alpha 2$ ) Gal $\beta 3$ GlcNAc $\beta 6$ (GalNAc $\alpha 3$ (Fuc $\alpha 2)$ Gal $\beta 3$ GlcNAc $\beta 3$ )Gal $\beta 3$ GlcNAc $\beta$ $3 \mathrm{Gal} \beta 4 \mathrm{Glc} \beta 1 \mathrm{Cer}), 4 \mu \mathrm{g}$. d Base peak chromatogram from LC-ESI/MS of the oligosaccharides obtained by digestion of fraction N2-IV from moose II small intestine with Rhodococcus endoglycoceramidase II. e Base peak chromatogram from LC-ESI/MS of the oligosaccharides obtained by digestion of fraction N2-VII from moose II small intestine with Rhodococcus endoglycoceramidase II. f Base peak chromatogram from LC-ESI/MS of the oligosaccharides obtained by digestion of fraction N2IX from moose II small intestine with Rhodococcus endoglycoceramidase II. The identification of individual glycosphingolipid-derived oligosaccharides given in D-F was based on their determined molecular masses and subsequent $\mathrm{MS}^{2}$ sequencing. A4, GalNAc $\alpha 3(\operatorname{Fuc} \alpha 2)$ Gal $\beta 4$ Glc; Gb3, Gal $\alpha 4$ Gal $\beta 4$ Glc; Gb4, GalNAc $\beta 3$ Gal $\alpha 4$ Gal $\beta 4 G l c ; ~ H 3$, Fuc $\alpha 2$ Gal $\beta 4$ Glc; nLc4, Gal $\beta 4$ GlcNAc $\beta 3$ Gal $\beta 4$ Glc; A6-1, GalNAc $\alpha 3$ (Fuc $\alpha 2)$ Gal $\beta 3$ GlcNAc $\beta 3$ Gal $\beta 4$ Glc; A8-1, GalNAc $\alpha 3(F u c \alpha 2)$ Gal $\beta 3$ GlcNAc $\beta 3$ Gal $\beta 3$ GlcNAc $\beta 3$ Gal $\beta 4$ Glc; B5, Gal $\alpha 3$ Gal $\beta$ 4 GlcNAc $\beta 3$ Gal $\beta 4$ Glc; B7, Gal $\alpha 3$ Gal $\beta 4$ GlcNAc $\beta 3$ Gal $\beta 4$ GlcNAc $\beta$ 3 Gal $\beta 4$ Glc; A12, GalNAc $\alpha 3$ (Fuc $\alpha 2$ ) Gal $\beta 3$ GlcNAc $\beta 6$ (GalNAc $\alpha 3$ (Fuc $\alpha 2)$ Gal $\beta 3$ GlcNAc $\beta 3$ )Gal $\beta 3$ GlcNAc $\beta 3$ Gal $\beta 4$ Glc. g $\mathrm{MS}^{2}$ of the ion at $m / z 1067$ (retention time $29.8 \mathrm{~min}$ ) from LC-ESI/MS of fraction N2-IX. h $\mathrm{MS}^{3}$ spectrum of the ion at $m / z$ 1607. The interpretation formula shows the deduced carbohydrate sequence

In summary, four glycosphingolipids with terminal blood group A determinants (A tetraosylceramide, A type 1 hexaosylceramide, A type 1 octaosylceramide, and A dodecaosylceramide) were tentatively identified by chromatogram binding assays and mass spectrometry.

\section{Characterization of the acid glycosphingolipids of moose I small intestine}

Chromatogram binding assays Probing of the total acid glycosphingolipid fractions from the moose intestines with monoclonal anti-GD1 a antibodies gave binding to all fractions (Fig. 8b). Also the monoclonal antibody directed against the SSEA-4 epitope gave a distinct binding to all fractions (Fig. 8c).

Binding of anti-SSEA-4 and anti-GD1a antibodies to the acid subfractions isolated from the small intestine of moose I demonstrated that the main anti-GD1a binding activity was to fractions A-V - A-VII (Fig. 8e), while the anti-SSEA-4 antibodies mainly bound to fractions A-IV - A-VI (Fig. 8f).
Mass spectrometry of the acid glycosphingolipids from moose I small intestine The base peak chromatogram obtained by LC-ESI/MS of the total acid glycosphingolipid fraction from moose I small intestine (Supplementary Figure 2A) was dominated by molecular ions at $\mathrm{m} / \mathrm{z} 794$ and $\mathrm{m} / \mathrm{z} 924$, indicating sulfatide with d18:1-h16:0 and t18:0-h24:0 ceramide, respectively. $\mathrm{MS}^{2}$ of these molecular ions gave a $\mathrm{B}_{1}$ ion at $\mathrm{m} / \mathrm{z}$ 241 and a $\mathrm{C}_{1}$ ion at $m / z 259$, confirming a $\mathrm{SO}_{3}$-Hex terminal (exemplified in Supplementary Figure 2B). Both $\mathrm{MS}^{2}$ spectra also had ions at $m / z 540$ and $m / z 522$, which are due to loss of the fatty acyl from the molecular ion [17]. The base peak chromatogram also had a minor molecular ion at $\mathrm{m} / z 1151$. $\mathrm{MS}^{2}$ of this ion gave a series of $\mathrm{Y}$ ions $\left(\mathrm{Y}_{0}\right.$ at $m / z 536, \mathrm{Y}_{1}$ at $\mathrm{m} / z$ 698, and $\mathrm{Y}_{2}$ at $\mathrm{m} / \mathrm{z} 860$ ), demonstrating a glycosphingolipid with NeuAc-Hex-Hex carbohydrate sequence and d18:1-16:0 ceramide, as the NeuAc-GM3 ganglioside (Supplementary Figure 2C). However, no other gangliosides could be identified by analysis of the total acid glycosphingolipid fraction.

Thus, in order to obtain sequence information of the slowmigrating gangliosides of moose I small intestine, the total acid glycosphingolipid fraction was separated into seven subfractions. LC-ESI/MS and $\mathrm{MS}^{2}$ of fraction A-I identified sulfatide with d18:1-h16:0 and t18:0-h24:0, as above, while fraction A-II had two predominant molecular ions at $\mathrm{m} / \mathrm{z} 1286$ and $m / z$ 1314. NeuGc-GM3 with t18:0-h22:0 and t18:0-h24:0 was identified by $\mathrm{MS}^{2}$ of the latter two ions (data not shown).

Fractions A-III and A-VII The base peak chromatograms from LC-ESI/MS of fractions A-III and A-VII (Supplementary Figure $3 \mathrm{~A}$ and $\mathrm{B}$ ) both had a doubly charged molecular ion at $\mathrm{m} / \mathrm{z}$ 917, eluting at 26.7-32.3 $\mathrm{min}$ and 27.9-31.9 min, respectively. This indicated gangliosides with two NeuAc, one HexNAc, three Hex, and d18:1-18:0 ceramide, as the GD1a or GD1b gangliosides. The $\mathrm{MS}^{2}$ spectra obtained were in both cases relatively weak. However, $\mathrm{MS}^{2}$ of the ion at $\mathrm{m} / \mathrm{z} 917$ of fraction A-III (Supplementary Figure 3C) gave a $\mathrm{B}_{2}$ ion at $\mathrm{m} / \mathrm{z}$ 581, demonstrating a NeuAc-NeuAc sequence, and a series of $\mathrm{Y}$ ions $\left(\mathrm{Y}_{1}\right.$ at $m / z 726, \mathrm{Y}_{2}$ at $m / z 888, \mathrm{Y}_{3 \alpha}$ at $m / z 1091$, and $\mathrm{Y}_{3 \beta}$ at $m / z$ 1254). Taken together this demonstrated a ganglioside with Hex-HexNAc-(NeuAc-NeuAc-)Hex-Hex sequence, as GD1b [18]. This conclusion was supported by the absence of binding of the anti-GD1a antibodies to fraction A-III (Fig. 8e, lane 2).

In contrast, a terminal NeuAc-Hex-HexNAc sequence was demonstrated by the $\mathrm{C}_{2 \alpha}$ ion at $m / z 470$ and the $\mathrm{B}_{3 \alpha}$ ion at $m / z$ 655 in the $\mathrm{MS}^{2}$ spectrum obtained of the ion at $\mathrm{m} / z 917$ of fraction A-VII (Supplementary Figure 3D). Taken together with the binding of the anti-GD1a antibodies to fraction A-VII (Fig. 8e, lane 6), this gave a tentative identification of a ganglioside with NeuAc-Hex-HexNAc(NeuAc)HexHex sequence and d18:1-18:0 ceramide, i.e., the GD1a ganglioside [18]. 
a Anisaldehyde detection

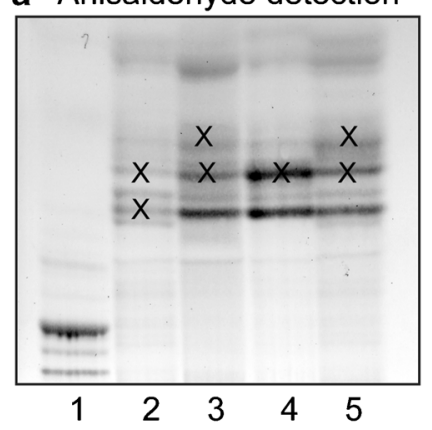

c Anti-SSEA-4

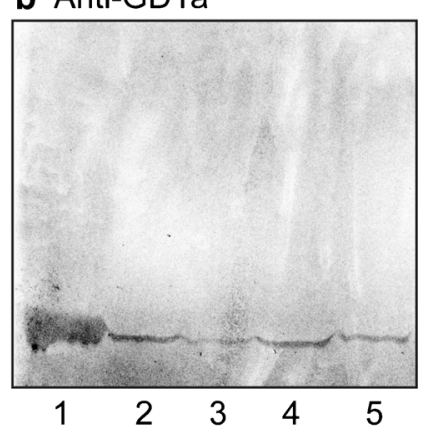

\section{c Anti-SSEA}

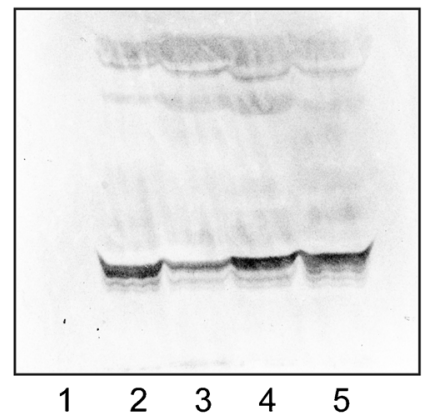

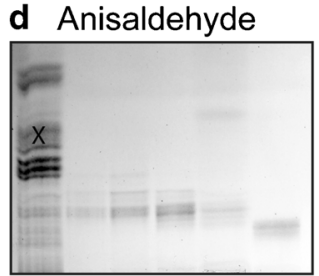

$1 \quad 2 \quad 3 \quad 4 \quad 556$ e Anti-GD1a

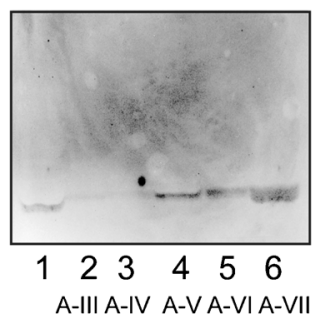

Fig. 8 Binding of monoclonal antibodies to the acid glycosphingolipids from moose small and large intestine. Thin-layer chromatogram after detection with anisaldehyde (a and $\mathbf{d}$ ), and autoradiograms obtained by binding of antibodies directed against the GD1 a ganglioside (b and e), the sialyl-globopenta/SSEA-4 determinant (c and f) and the GM2 ganglioside (g). The glycosphingolipids were separated on aluminum-backed silica gel plates, using chloroform/methanol/water 60:35:8 (by volume) as solvent system, and the binding assays were performed as described under "Materials and methods". Autoradiography was for $12 \mathrm{~h}$. The

Fractions A-IV - A-VI The base peak chromatograms of fractions A-IV, A-V and A-VI showed that these three fractions all contained several compounds (Fig. 9). Both fractions A-IV and A-V had doubly charged molecular ions at $m / z 721$ and $m / z 763$, both corresponding to a ganglioside with two NeuAc and two Hex, having d18:1-16:0 and d18:1-22:0 ceramide, respectively. $\mathrm{MS}^{2}$ of the molecular ion at $m / z 721$ (data not shown) gave two $\mathrm{B}$ ions ( $\mathrm{B}_{1}$ at $m / z 290$ and $\mathrm{B}_{2}$ at $m / z 581$ ) demonstrating a terminal NeuAc-NeuAc sequence. In addition, there was a series of $\mathrm{Y}$ ions $\left(\mathrm{Y}_{0}\right.$ at $\mathrm{m} / \mathrm{z} 536, \mathrm{Y}_{2}$ at $\mathrm{m} / \mathrm{z}$

f Anti-SSEA-4

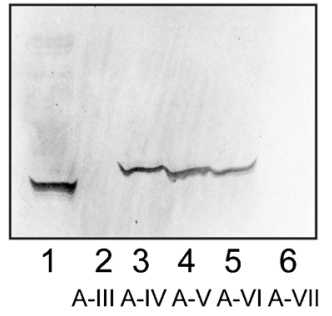

lanes on a-c were: Lane 1, calf brain gangliosides, $40 \mu \mathrm{g}$; Lane 2, total acid glycosphingolipids of moose I small intestine, $40 \mu \mathrm{g}$; Lane 3, total acid glycosphingolipids of moose II small intestine, $40 \mu \mathrm{g}$; Lane 4 , total acid glycosphingolipids of moose III small intestine, $40 \mu \mathrm{g}$; Lane 5, total acid glycosphingolipids of moose I large intestine, $40 \mu \mathrm{g}$. The lanes on dg were: Lane 1, total acid glycosphingolipids of moose I small intestine, $40 \mu \mathrm{g}$; Lanes 2-6, fractions A-III - A-VII, $4 \mu \mathrm{g} /$ lane. The bands marked with $\mathrm{X}$ on (a) and (d) are non-glycosphingolipid contaminants

860 , and $Y_{3}$ at $m / z$ 1151). Thus, a ganglioside with NeuAcNeuAc-Hex-Hex sequence and d18:1-16:0 ceramide, as the NeuAc-GD3 ganglioside, was indicated. $\mathrm{MS}^{2}$ of the doubly charged molecular ion at $\mathrm{m} / \mathrm{z} 763$ also identified a NeuAcGD3 ganglioside, in this case with d18:1-22:0 ceramide (data not shown).

The doubly charged molecular ion at $m / z 853$ in fraction AIV indicated a ganglioside with one NeuAc, one HexNAc, four Hex, and d18:1-18:0 ceramide, while the doubly charged molecular ion at $m / z 847$ in fractions A-V and A-VI indicated a

Fig. 9 LC-ESI/MS of the acid glycosphingolipids from moose I small intestine. a Base peak chromatogram from LC-ESI/MS of fraction A-IV from moose I small intestine. b Base peak chromatogram from LC-ESI/MS of fraction A-V from moose I small intestine. c Base peak chromatogram from LC-ESI/MS of fraction A-VI from moose I small intestine

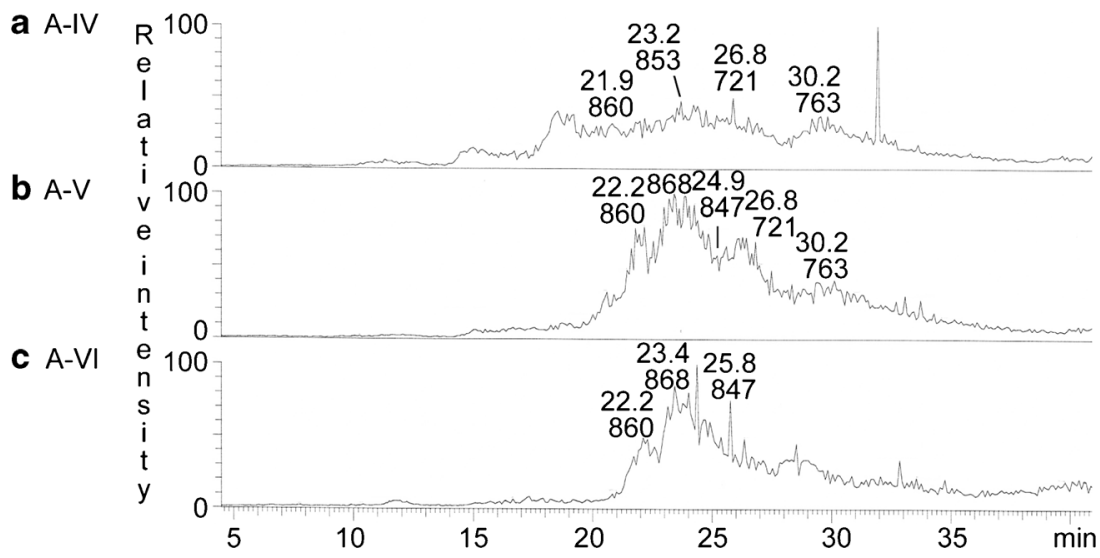


ganglioside with one NeuGc, one HexNAc, four Hex, and d18:1-16:0 ceramide. $\mathrm{MS}^{2}$ of the ion at $m / z 853$ (Fig. 10a) gave $B$ and $C$ type fragment ions $\left(\mathrm{B}_{1}\right.$ at $m / z 290, \mathrm{C}_{1}$ at $m / z 308$, $\mathrm{C}_{2}$ at $m / z 470, \mathrm{~B}_{3}$ at $m / z 655, \mathrm{C}_{3}$ at $m / z 673, \mathrm{~B}_{4}$ at $m / z 817, \mathrm{~B}_{5}$ at $m / z 979$, and $\mathrm{C}_{5}$ at $m / z$ 997) demonstrating a NeuAc-HexHexNAc-Hex-Hex-Hex carbohydrate sequence. The $\mathrm{Y}_{3}$ ion at $m / z 1050$ was further evidence for a Hex-Hex-Hex sequence at the reducing end, and the ${ }^{0,2} \mathrm{~A}_{5}$ ion at $m / z 937$ indicated a 4substitution of the second Hex from the reducing end.

The same carbohydrate sequence but with NeuGc instead of NeuAc, i.e., a NeuGc-Hex-HexNAc-Hex-Hex-Hex sequence, was indicated by $\mathrm{MS}^{2}$ of the molecular ion at $\mathrm{m} / \mathrm{z}$ 847 in fraction A-V and A-VI (Fig. 10b). This was concluded from the $\mathrm{B}$ and $\mathrm{C}$ type fragment ions series ( $\mathrm{B}_{1}$ at $m / z 306, \mathrm{C}_{1}$ at $m / z 324, \mathrm{C}_{2}$ at $m / z 486, \mathrm{~B}_{3}$ at $m / z 671, \mathrm{C}_{3}$ at $m / z 689, \mathrm{C}_{4}$ at $\mathrm{m} / \mathrm{z} 851, \mathrm{~B}_{5}$ at $\mathrm{m} / \mathrm{z} 995$, and $\mathrm{C}_{5}$ at $\mathrm{m} / \mathrm{z}$ 1013). Here, the $\mathrm{Y}_{3}$ ion at $m / z 1022$ was evidence for a Hex-Hex-Hex sequence at the reducing end, and the ${ }^{0,2} \mathrm{~A}_{5}$ ion at $\mathrm{m} / \mathrm{z} 953$ indicated that the second Hex from the reducing end was substituted at C-4.

Thus, $\mathrm{MS}^{2}$ indicated NeuAc and NeuGc variants of a ganglioside with NeuAc/NeuGc-Hex-HexNAc-Hex-Hex-Hex sequence, most likely sialyl-globopentaosylceramide (SSEA-4 ganglioside). This suggestion was corroborated by the binding of anti-sialyl-globopenta/SSEA-4 antibodies to fractions AIV, A-V and A-VI (Fig. 8f).

The doubly charged molecular ions at $m / z 860$ in fractions A-IV - A-VI indicated a ganglioside with one NeuAc, two HexNAc, and three Hex, and d18:1-16:0, while the molecular ion at $m / z 868$ fractions A-V and A-IV suggested a ganglioside with one NeuGc, two HexNAc, and three Hex, and d18:116:0.

The $\mathrm{MS}^{2}$ spectrum of the ion at $m / z 860$ (Fig. 10c) had a number of B- and C-type fragment ions $\left(\mathrm{B}_{1}\right.$ at $m / z 290, \mathrm{C}_{2}$ at $m / z 673, \mathrm{C}_{3}$ at $m / z 876, \mathrm{~B}_{5}$ at $m / z 1020, \mathrm{C}_{4}$ at $m / z 1038$, and $\mathrm{B}_{5}$
Fig. 10 LC-ESI/MS of the acid glycosphingolipid fractions from moose I small intestine. a $\mathrm{MS}^{2}$ of the ion at $m / z 853$ (retention time $23.9 \mathrm{~min}$ ) from LC-ESI/MS of fraction A-IV. $\mathbf{b ~ M S}{ }^{2}$ of the ion at $\mathrm{m} / \mathrm{z} 847$ (retention time $25.0 \mathrm{~min}$ ) from LC-ESI/MS of fraction AVI. c $\mathrm{MS}^{2}$ of the ion at $\mathrm{m} / \mathrm{z} 860$ (retention time $22.0 \mathrm{~min}$ ) from LC-ESI/MS of fraction A-V. d $\mathrm{MS}^{2}$ of the ion at $m / z 868$ (retention time $23.7 \mathrm{~min}$ ) from LC-ESI/MS of fraction A-V. e Interpretation formulas showing the deduced carbohydrate sequences and ceramide composition

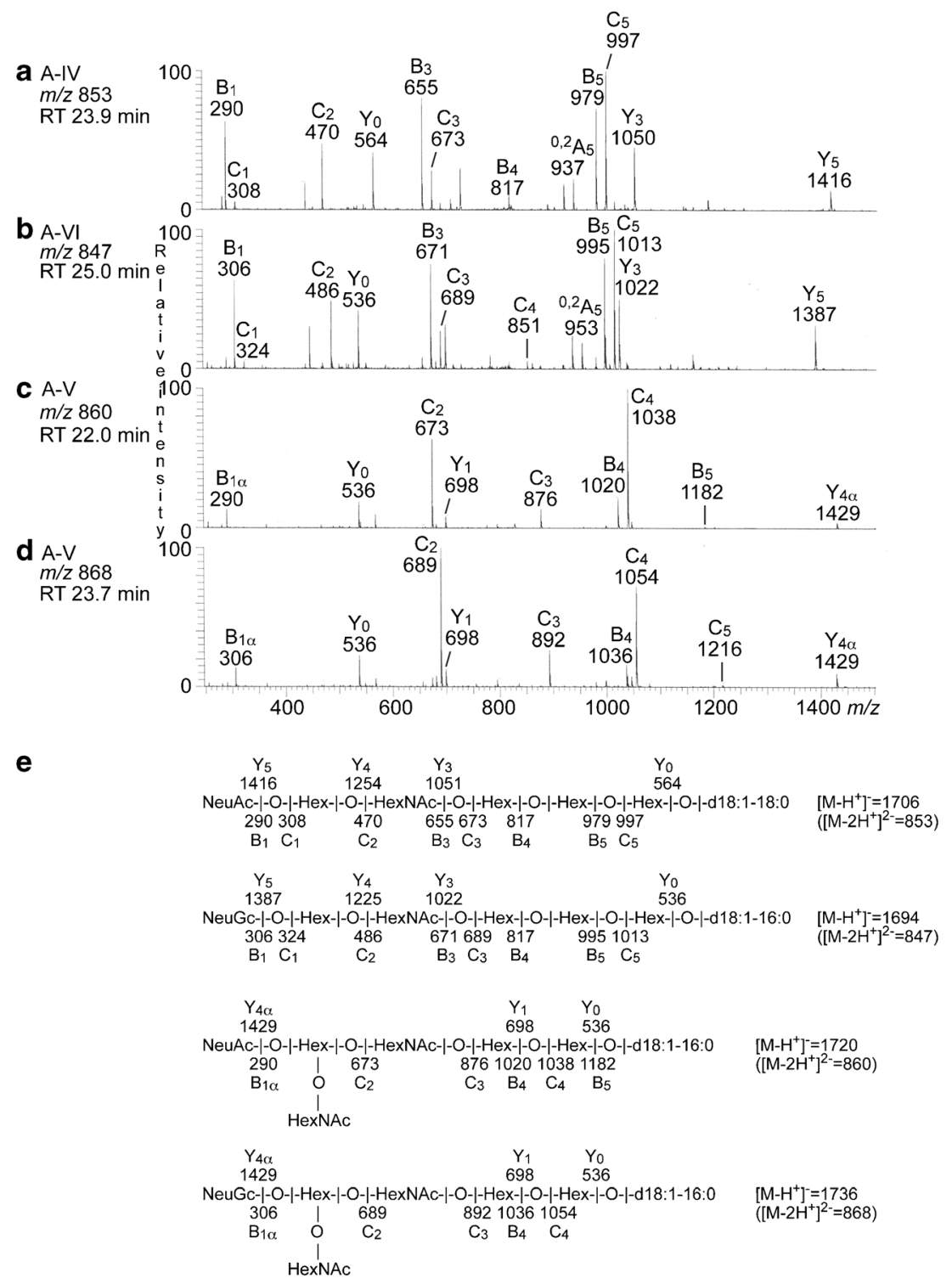


at $m / z$ 1182) demonstrating a NeuAc-(HexNAc+Hex)HexNAc-Hex-Hex sequence, and the $\mathrm{MS}^{2}$ spectrum of the ion at $\mathrm{m} / z 868$ (Fig. 10d) had the corresponding B- and Ctype fragment ions $\left(\mathrm{B}_{1}\right.$ at $\mathrm{m} / z 306, \mathrm{C}_{2}$ at $\mathrm{m} / \mathrm{z} 689, \mathrm{C}_{3}$ at $\mathrm{m} / \mathrm{z}$ $892, \mathrm{C}_{4}$ at $\mathrm{m} / \mathrm{z} 1054$, and $\mathrm{C}_{5}$ at $\mathrm{m} / \mathrm{z} 1216$ ) demonstrating a NeuGc-(HexNAc+Hex)-HexNAc-Hex-Hex sequence. No $\mathrm{B}_{2}$ or $\mathrm{C}_{2}$ ions were obtained by $\mathrm{MS}^{2}$ or $\mathrm{MS}^{3}$, so the order of the penultimate HexNAc and Hex could not be determined.

Thus, $\mathrm{MS}^{2}$ of the molecular ions at $\mathrm{m} / \mathrm{z} 860$ and $\mathrm{m} / \mathrm{z}$ 868 demonstrated NeuAc and NeuGc variants of a ganglioside with one NeuAc or NeuGc, two HexNAc and three Hex. Of previously identified gangliosides there are three alternatives. Firstly, the GalNAc-GM1 ganglioside (GalNAc $\beta 3$ Gal $\beta 3$ GalNAc $\beta 4$ (NeuAc/NeuGc $\alpha 3$ ) $\mathrm{Gal} \beta 4 \mathrm{Glc} \beta 1 \mathrm{Cer})$. However, this alternative is not likely since the $\mathrm{C}_{3}$ ions at $\mathrm{m} / \mathrm{z} 673$ and $\mathrm{m} / \mathrm{z} 689$ demonstrated a terminal NeuAc-Hex-HexNAc sequence and a terminal NeuGc-Hex-HexNAc sequence, respectively. The second alternative is the NeuAc- $x_{2}$ ganglioside (NeuAc $\alpha$ $3 \mathrm{GalNAc} \beta 3 \mathrm{Gal} \beta 4 \mathrm{GlcNAc} \beta 3 \mathrm{Gal} \beta 4 \mathrm{Glc} \beta 1 \mathrm{Cer})$ and the third is the $\mathrm{Sd}^{\mathrm{a}}$ ganglioside (NeuAc $\alpha 3(\mathrm{GalNAc} \beta 4)$ Gal $\beta 4$ GlcNAc $\beta 3$ Gal $\beta 4$ Glc $\beta 1$ Cer). $\mathrm{MS}^{3}$ gave no $\mathrm{B}_{2}$ or $\mathrm{C}_{2}$ ions that allowed differention between these two alternatives. However, the binding of anti-GM2 antibodies in the slowmigrating region of fractions A-IV - A-VI (Fig. 8g) supported a $\mathrm{Sd}^{\mathrm{a}}$ ganglioside, since these antibodies recognize a terminal NeuAc $\alpha 3($ GalNAc $\beta 4)$ Gal sequence.

Comparison with fallow deer intestine Acid and non-acid glycosphingolipids were also isolated from mucosal scrapings of fallow deer small intestine, with the intention of comparing with the intestinal glycosphingolipid profile of a species related to the moose. The major compound of the non-acid fraction of fallow deer intestine was monoglycosylceramide. By separation into subfractions, and characterization of these with chromatogram binding assays and mass spectrometry as above, the two major complex non-acid glycosphingolipids were characterized as Galili penta- and heptaosylceramide (data not shown). However, no glycosphingolipids with terminal blood group $\mathrm{A} / \mathrm{B} / \mathrm{H}$ determinants were found.

The acid glycosphingolipid fractions of moose small intestine and fallow deer small intestine were very similar. Thus, sulfatide, and the gangliosides GM3, GD3, GD1a and GD1b, were also characterized in the case of fallow deer intestine, using the same strategies as for moose intestine (data not shown).

Furthermore, a subfraction with distinct binding of antiSSEA-4 antibodies was obtained upon separation of the acid fraction from fallow deer intestine (Fig. 11b, lane 4). LC-ESI/ MS of this fraction gave a base peak chromatogram with a number of doubly charged molecular ions at $m / z 763, \mathrm{~m} / \mathrm{z} 860$, $\mathrm{m} / \mathrm{z} 881, \mathrm{~m} / \mathrm{z} 901$ and $\mathrm{m} / \mathrm{z} 909$ (Fig. 11c). The doubly charged molecular ion at $\mathrm{m} / \mathrm{z} 881$ indicated a ganglioside with one
NeuAc, one HexNAc, four Hex, and d18:1-22:0 ceramide. A NeuAc-Hex-HexNAc-Hex-Hex-Hex carbohydrate sequence was demonstrated by the $\mathrm{B}$ and $\mathrm{C}$ type fragment ions $\left(\mathrm{B}_{1}\right.$ at $m / z 290, \mathrm{C}_{2}$ at $m / z 470, \mathrm{~B}_{3}$ at $m / z 655, \mathrm{C}_{3}$ at $m / z 673, \mathrm{C}_{4}$ at $m / z 835, \mathrm{~B}_{5}$ at $m / z 979, \mathrm{C}_{5}$ at $m / z$ 997, and $\mathrm{C}_{6}$ at $\mathrm{m} / z$ 1159) obtained by $\mathrm{MS}^{2}$ (Fig. 11d). The features of this $\mathrm{MS}^{2}$ spectrum were very similar to the $\mathrm{MS}^{2}$ spectrum of moose intestinal sialyl-globopentaosylceramide/SSEA-4 (Fig. 10a), allowing identification of this ganglioside also in fallow deer intestine.

In a similar manner, a NeuAc-Sd ${ }^{\text {a }}$ ganglioside with d18:122:0 ceramide was identified by $\mathrm{MS}^{2}$ of the ion at $\mathrm{m} / \mathrm{z} 902$ (Fig. 11e). This was concluded from the series of $B$ and $C$ type fragment ions obtained by $\mathrm{MS}^{2}\left(\mathrm{~B}_{1}\right.$ at $m / z 290, \mathrm{C}_{2}$ at $m / z 673$, $\mathrm{B}_{3}$ at $m / z$ 858, $\mathrm{B}_{5}$ at $m / z 1020, \mathrm{C}_{4}$ at $m / z 1038$, and $\mathrm{B}_{5}$ at $m / z$ 1182) demonstrating a NeuAc-(HexNAc $+\mathrm{Hex})$-HexNAcHex-Hex sequence. Furthermore, a NeuAc-Sd ${ }^{a}$ ganglioside with d18:1-16:0 ceramide was found by $\mathrm{MS}^{2}$ of the ion at $\mathrm{m} / \mathrm{z} 860$, and a NeuGc-Sd ${ }^{\mathrm{a}}$ ganglioside with d18:1-22:0 ceramide found by $\mathrm{MS}^{2}$ of the ion at $\mathrm{m} / z$ 909. Finally, $\mathrm{MS}^{2}$ of the ion at $\mathrm{m} / \mathrm{z} 763$ identified a NeuAc-GD3 ganglioside with d18:1-22:0 ceramide (data not shown).

\section{Discussion}

Moose are the largest land mammals in the circumpolar boreal forests of Eurasia and Canada, and are an important game species. Their diet consists of browse, tree, and shrub leaves and stems, and like all ruminants, the moose gastrointestinal tract has a four chambered stomach, in addition to the small and large intestine. In this study the non-acid and acid glycosphingolipids of moose small and large intestine were characterized. The complex non-acid glycosphingolipid fractions of the three small intestines, and the one large intestine, all had Galili penta- and heptaglycosylceramides. In addition, two of the moose small intestines, and the one large intestine, had glycosphingolipids with terminal blood group $\mathrm{O}$ determinants (H triglycosylceramide, $\mathrm{H}$ type 2 pentaosylceramide, $\mathrm{H}$ type 1 penta- and heptaosylceramide), while glycosphingolipids with terminal blood group A determinants (A tetraosylceramide, A type 1 hexa- and octaosylceramide, A dodecaosylceramide), were found in the third moose small intestine.

Glycosphingolipids with terminal histo-blood group A, B or $\mathrm{O}$ determinants are present in the gastrointestinal tract of many different species [2]. The types of ABO determinants varies depending on species, and also between individuals of the same species. Thus, in the human small intestinal epithelium the expression of glycosphingolipids with terminal blood group $\mathrm{A}, \mathrm{B}$ or $\mathrm{O}$ determinants varies depending on the ABO blood group of the individual donors [19]. However, in pigs there are only blood group $\mathrm{A}$ and $\mathrm{O}$ determinants [20]. Here, the $\mathrm{H}$ type 1 pentaglycosylceramide is present 


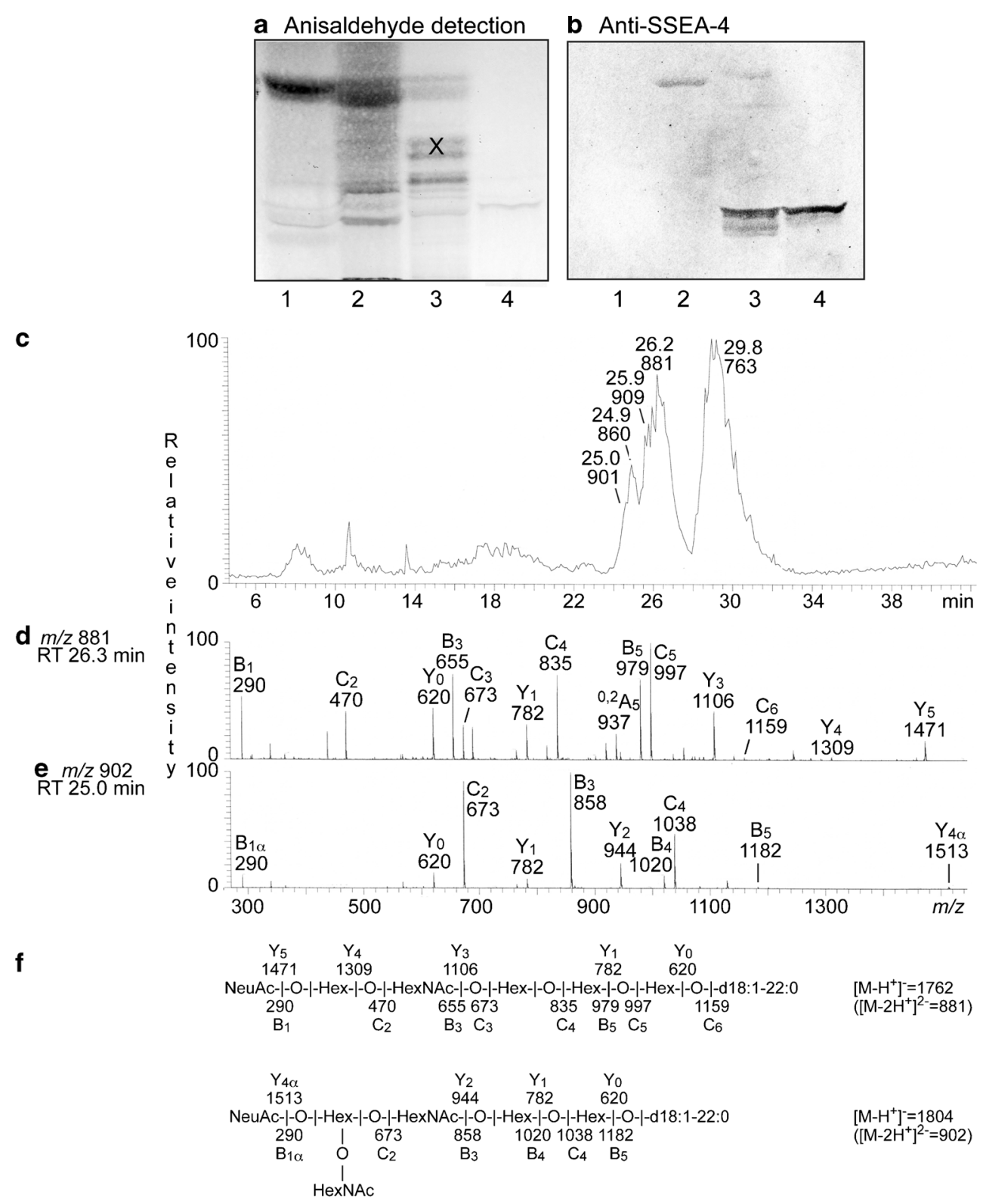

Fig. 11 Comparison of acid glycosphingolipids from horse, bovine, and moose small intestine. Thin-layer chromatogram after detection with anisaldehyde (a), and autoradiogram obtained by binding antibodies directed against the sialyl-globopenta/SSEA-4 determinant (b). The glycosphingolipids were separated on aluminum-backed silica gel plates, using chloroform/methanol/water 60:35:8 (by volume) as solvent system, and the binding assays were performed as described under "Materials and methods". Autoradiography was for $12 \mathrm{~h}$. The lanes were: Lane 1, total acid glycosphingolipids of horse small intestine, $40 \mu \mathrm{g}$; Lane 2, total acid glycosphingolipids of bovine small intestine,

in the small intestinal epithelium of blood group $\mathrm{O}$ pigs, while blood group A pigs have the A type 1 hexaglycosylceramide, the A type 4 heptaglycosylceramide, the A type 1 octaglycosylceramide, and a repetitive A type 1 nonaglycosylceramide [21].

In this study we demonstrate that glycosphingolipids with terminal blood group $\mathrm{H}$ determinants are present in
$40 \mu \mathrm{g}$; Lane 3, total acid glycosphingolipids of moose small intestine, $40 \mu \mathrm{g}$; Lane 1, acid glycosphingolipid subfraction (fraction FD-VI) isolated from fallow deer small intestine, $4 \mu \mathrm{g}$. The bands marked with an $\mathrm{X}$ in lane 3 are non-glycosphingolipid contaminants. c Base peak chromatogram from LC-ESI/MS of fraction fraction FD-VI from fallow deer small intestine. $\mathbf{d ~ M S ^ { 2 }}$ of the ion at $\mathrm{m} / \mathrm{z} 881$ (retention time $26.3 \mathrm{~min}$ ) from LC-ESI/MS of fraction FD-VI. e MS ${ }^{2}$ of the ion at $\mathrm{m} / z 902$ (retention time $25.0 \mathrm{~min}$ ) from LC-ESI/MS of fraction FD-VI. f Interpretation formulas showing the deduced carbohydrate sequences and ceramide composition

the small intestine of certain moose individuals, whereas other moose individuals have glycosphingolipids with terminal blood group A determinants. However, due to the small number of moose intestines characterized, it can not be excluded that there are also moose with intestinal glycosphingolipids with blood group B determinants. 
In addition to the glycosphingolipids with blood group determinant, all the intestinal samples had glycosphingolipids belonging to the lacto- and neolactoseries (lactotriaosylceramide, lactotetraosylceramide, neolactotetraosylceramide, Gal $\alpha 3-\mathrm{Le}^{\mathrm{x}}$ hexaosylceramide, and lacto-neolactohexaosylceramide), globoseries (globotriaosylceramide and globotetraosylceramide), and isogloboseries (isoglobotriaosylceramide). Thus, moose intestine is a good source for isolation of variant glycosphingolipids.

The chemical staining of the total non-acid fractions separated on thin-layer chromatograms shows that the tri- and tetraglycosylceramides are major compounds. Yet, in the base peak chromatograms from LC/ESI-MS of the oligosaccharides released from the glycosphingolipids, the molecular ions corresponding to tri- and tetrasaccharides (at $m / z 503,544$ and 706) are relatively minor. This discrepancy is due to the low capacity of the Rhodococcus endoglycoceramidase for hydrolysis of globo-series glycosphingolipids [22, 23]. The ideal enzyme would have been the ceramide glycanase from Macrobdella decora, which has a more universal hydrolytic activity towards glycosphingolipids [24]. However, this enzyme is no longer available on the market. In fact, in certain cases, as when analyzing total non-acid glycosphingolipid mixtures with high amounts of globotri- and globotetraosylceramide, this relative resistance Rhodococcus endoglycoceramidase might be an advantage allowing detection of minor complex glycosphingolipids.

Acid fractions of moose small and large intestine were very similar, i.e., all contained sulfatide, and the gangliosides GM3, GD3, GD1a, GD1b, and also the Sd $\mathrm{d}^{\mathrm{a}}$ ganglioside and sialylglobopentaosylceramide/SSEA-4. Interestingly, the same acid glycosphingolipids were present in fallow deer small intestine. However, no binding of the anti-SSEA-4 antibodies to the acid glycosphingolipids of horse and bovine small intestine occurred, indicating that sialyl-globopentaosylceramide was not present in these species. This is consistent with previous characterization of the acid glycosphingolipids of adult bovine small intestinal epithelium were sulfatide, NeuAc-/NeuGcGM3, NeuAc-/NeuGc-GM2, NeuAc-/NeuGc-GM1, and GcGD2 were found [25].

The $\mathrm{Sd}^{\text {a }}$ determinant is expressed on epithelial glycosphingolipids and glycoproteins, and mucins in the normal gastrointestinal tract in the majority of humans, and in several other species [26]. The structure of the $\mathrm{Sd}^{\mathrm{a}}$ determinant was first determined as the GalNAc $\beta 4(\mathrm{NeuAc} \alpha 3) \mathrm{Gal} \beta 4 \mathrm{GlcNAc} \beta 3 \mathrm{Gal}$ pentasaccharide carried by $\mathrm{N}$-glycans of the Tamm-Horsfall glycoprotein [27]. The $\mathrm{Sd}^{\mathrm{a}}$ antigen is related to, but not entirely identical with, the Cad antigen. The Cad antigen was first characterized as the O-linked pentasaccharide GalNAc $\beta 4($ NeuAc $\alpha 3$ )Gal $\beta 3$ (NeuAc $\alpha 6$ )GalNAc of glycophorin A [28]. Thus, the two antigens have the same terminal trisaccharide, GalNAc $\beta 4(\mathrm{Neu} A c \alpha 3) \mathrm{Gal} \beta$. Interestingly, a number of different physiological and pathological roles of the $\mathrm{Sd}^{\mathrm{a}}$ determinant have been described (reviewed in [29]). Thus, in the murine system the $\mathrm{Sd}^{\mathrm{a}}$ antigen plays a role in the lytic function of cytotoxic T lymfocytes, is involved in the regulation of the circulating half-life of the von Willebrand factor and also in implantation of embryos to the endometrium. Furthermore, overexpression of the $\mathrm{Sd}^{\mathrm{a}}$ determinant in skeletal muscle fibers gives a reversion of dystrophy in murine models of muscular dystrophy. Finally, in humans the $\mathrm{Sd}^{\mathrm{a}}$ antigen is down-regulated in gastric and colon cancer.

The finding of sialyl-globopentaosylceramide/SSEA-4, with both $\mathrm{N}$-acetyl and $\mathrm{N}$-glycolyl neuraminic acid, in moose intestine is to our knowledge the first time sialylglobopentaosylceramide/SSEA-4 has been characterized in a normal fully differentiated tissue, and also the first time NeuGc-globopentaosylceramide has been characterized. NeuAc-globopentaosylceramide carries the determinant of the stage specific embryonic antigen 4 (SSEA-4). The stage specific embryonic antigens were originally identified by three monoclonal antibodies (SSEA-1, SSEA-3, and SSEA4) prepared against murine embryos [30], and recognize defined carbohydrate epitopes associated with the lacto- and globo-series glycosphingolipids [31-33]. In humans SSEA-4 is a marker for embryonic and adult stem cells [34-36]. Upon differentiation of human embryonic stem cells, the expression of SSEA-4 is rapidly diminished, and has almost completely disappeared after 14 days [37, 38], i.e., SSEA-4 downregulation accompanies the loss of pluripotency.

According to the cancer stem cells theory [39], cancers are maintained by subpopulations of tumor cells with stem or progenitor cell characteristics expressing stem cell associated markers, and it are these cells that initiate tumor formation and differentiate along multi-potent pathways. In agreement with this theory, SSEA-4 is also expressed in several human cancers, as e.g., breast cancer [40], ovarian carcinoma [41], renal cell carcinoma [42], basaloid lung cancer [43], and glioblastoma [44].

However, in the present study the sialyl-globopenta/SSEA4 ganglioside was characterized in moose small and large intestine, and also in fallow deer small intestine, i.e., in normal tissues of adult individuals. This once again demonstrates that the one carbohydrate structure may have different biological roles in different species, at different times in development and in different tissues.

Acknowledgments The authors would like to acknowledge Eva and Carl-Otto Dahlén for collecting the moose intestinal tissues. This study was supported by the Swedish Medical Research Council (Grant No. 12628), the Swedish Cancer Foundation, and governmental grants to the Sahlgrenska University Hospital. The use of the LTQ linear quadrupole ion trap mass spectrometer (obtained by a grant from the Swedish Research Council (No. 342-2004-4434) to Gunnar Hansson), is gratefully acknowledged.

The glycosphingolipid nomenclature follows the recommendations by the IUPAC-IUB Commission on Biochemical Nomenclature (CBN for Lipids: Eur. J. Biochem. (1998) 257, 293). It is assumed 
that Gal, Glc, GlcNAc, and GalNAc, are of the D-configuration, Fuc of the L-configuration, and all sugars present in the pyranose form.

Open Access This article is distributed under the terms of the Creative Commons Attribution 4.0 International License (http:// creativecommons.org/licenses/by/4.0/), which permits unrestricted use, distribution, and reproduction in any medium, provided you give appropriate credit to the original author(s) and the source, provide a link to the Creative Commons license, and indicate if changes were made.

\section{References}

1. Breimer, M.E., Hansson, G.C., Karlsson, K.-A., Leffler, H.: Blood group type glycosphingolipids from the small intestine of different animals analysed by mass spectrometry and thin-layer chromatography. A note on species diversity. J. Biochem. 90, 589-609 (1981)

2. Hansson, G.C.: Structural aspects of blood group glycosphingolipids in the gastrointestinal tract. Adv. Exp. Med. Biol. 228, 465-494 (1988)

3. Breimer, M.E., Hansson, G.C., Karlsson, K.-A., Larson, G., Leffler, H.: Glycosphingolipid composition of epithelial cells isolated along the villus axis of small intestine of a single human individual. Glycobiology 22, 1721-1730 (2012)

4. Karlsson, K.-A.: Preparation of total non-acid glycolipids for overlay analysis of receptors for bacteria and viruses and for other studies. Meth. Enzymol. 138, 212-220 (1987)

5. Samuelsson, B.E., Pimlott, W., Karlsson, K.-A.: Mass spectrometry of mixtures of intact glycosphingolipids. Meth. Enzymol. 193, 623-646 (1990)

6. Karlsson, H., Halim, A., Teneberg, S.: Differentiation of glycosphingolipid-derived glycan structural isomers by liquid chromatography-mass spectrometry. Glycobiology 20, 1103-1116 (2010)

7. Koerner Jr., T.A.W., Prestegard, J.H., Demou, P.C., Yu, R.K.: Highresolution proton NMR studies of gangliosides. 1. Use of homonuclear two-dimensional spin-echo J-correlated spectroscopy for determination of residue composition and anomeric configurations. Biochemistry 22, 2676-2687 (1983)

8. Waldi, D.: Sprühreagentien für die dünnschicht-chromatographie. In: Stahl, E. (ed.) Dünnschicht-Chromatographie, pp. 496-515. Springer, Berlin (1962)

9. Svennerholm, L., Fredman, P.: A procedure for the quantitative isolation of brain gangliosides. Biochim. Biophys. Acta 617, 97 109 (1980)

10. Hansson, G.C., Karlsson, K.-A., Larson, G., McKibbin, J.M., Blaszczyk, M., Herlyn, M., Steplewski, Z., Koprowski, H.: Mouse monoclonal antibodies against human cancer cell lines with specificities for blood group and related antigens. Characterization by antibody binding to glycosphingolipids in a chromatogram binding assay. J. Biol. Chem. 258, 4091-4097 (1983)

11. Teneberg, S., Ångström, J., Jovall, P.-Å., Karlsson, K.-A.: Characterization of binding of Gal $\beta 4 \mathrm{GlcNAc}$-specific lectins from Erythrina christagalli and Erythrina corallodendron to glycosphingolipids. Detection, isolation and characteriztion of a novel glycosphingolipid of bovine buttermilk. J. Biol. Chem. 269, 8554-8563 (1994)

12. Benktander, J., Ångström, J., Breimer, M.E., Teneberg, S.: Redefinition of the carbohydrate binding specificity of Helicobacter pylori BabA adhesin. J. Biol. Chem. 287, 31712-31724 (2012)

13. Ångström, J., Teneberg, S., Abul Milh, M., Larsson, T., Leonardsson, I., Olsson, B.-M., Ölwegård Halvarsson, M.,
Danielsson, D., Näslund, I., Ljungh, Å., Wadström, T., Karlsson, K.-A.: The lactosylceramide binding specificity of Helicobacter pylori. Glycobiology 8, 297-309 (1998)

14. Chai, W., Piskarev, V., Lawson, A.M.: Negative-ion electrospray mass spectrometry of neutral underivatized oligosaccharides. Anal. Chem. 73, 651-657 (2001)

15. Bouhours, D., Liaigre, J., Naulet, J., Maume, D., Bouhours, J.F.: A novel glycosphingolipid expressed in pig kidney: Gal alpha 13Lewis(x) hexaglycosylceramide. Glycoconj. J. 14, 29-38 (1997)

16. Aspholm-Hurtig, M., Dailide, G., Lahmann, M., Kalia, A., Ilver, D., Roche, N., Vikström, S., Sjöström, R., Lindén, S., Bäckström, A., Arnqvist, A., Mahdavi, J., Nilsson, U.J., Velapatiño, B., Gilman, R.H., Gerhard, M., Alarcon, T., López-Brea, M., Nakazawa, T., Fox, J.G., Correa, P., Dominguez-Bello, M.G., Perez-Perez, G.I., Blaser, M.J., Normark, S., Carlstedt, I., Oscarson, S., Teneberg, S., Berg, D.E., Borén, T.: Functional adaptation of BabA, the Helicobacter pylori blood-group antigen binding adhesin. Science 305, 519-522 (2004)

17. Hsu, F.-F., Turk, J.: Studies on sulfatides by quadrupole ion-trap mass spectrometry with electrospray ionization: Structural characterization and the fragmentation processes that include an unusual internal galactose residue loss and the classical charge-remote fragmentation. J. Am. Soc. Mass Spectrom. 15, 536-546 (2004)

18. Ito, E., Tominaga, E., Waki, H., Miseki, K., Tomioka, A., Nakajima, K., Kakehi, K., Suzuki, M., Taniguchi, N., Suzuki, A.: Structural characterization of monosialo-, disialo- and trisialo-gangliosides by negative ion AP-MALDI-QIT-TOF mass spectrometry with $\mathrm{MS}^{\mathrm{n}}$ switching. Neurochem. Res. 37, 1315-1324 (2012)

19. Björk, S., Breimer, M.E., Hansson, G.C., Karlsson, K.-A., Leffler, H.: Structures of blood group glycosphingolipids of human small intestine. A relation between the expression of fucolipids of epithelial cells and the ABO, Le amnd Se phenotype of the donor. J. Biol. Chem. 262, 6758-6765 (1987)

20. Sako, F., Gasa, S., Makita, A., Hayashi, A., Nozawa, S.: Human blood group glycosphingolipids of porcine erythrocytes. Arch. Biochem. Biophys. 278, 228-237 (1990)

21. Coddens, A., Diswall, M., Ångström, J., Breimer, M.E., Goddeeris, B., Cox, E., Teneberg, S.: Recognition of blood group ABH type 1 determinants by the FedF adhesin of F18-fimbriated Escherichia coli. J. Biol. Chem. 284, 9713-9726 (2009)

22. Ito, M., Yamagata, T.: Purification and characterization of glycosphingolipid-specific endoglycosidases (endoglycoceramidases) from a mutant strain of Rhodococcus sp. Evidence for three molecular species of endoglycoceramidase with different specificities. J. Biol. Chem. 264, 9510-9519 (1989)

23. Li, Y.-T., Chou, C.-W., Li, S.-C., Kobayashi, U., Ishibashi, Y.-H., Ito, M.: Preparation of homogenous oligosaccharide chains from glycosphingolipids. Glycoconj. J. 26, 929-933 (2009)

24. Zhou, B., Li, S.-C., Laine, R.A., Huang, R.T.C., Li, Y.-T.: Isolation and characterization of ceramide glycanase from the leech, Macrodella decora. J. Biol. Chem. 264, 12272-12277 (1989)

25. Delorme, C., Brüssow, H., Sidoti, J., Roche, N., Karlsson, K.-A., Neeser, J.-R., Teneberg, S.: Glycosphingolipid binding specificities of rotavirus: Identification of a sialic acid binding epitope. J. Virol. 75, 2276-2287 (2001)

26. Morton, J.A., Pickles, M.M., Terry, A.M.: The $\mathrm{Sd}^{\text {a }}$ blood group antigen in tissues and body fluids. Vox Sang. 19, 472-482 (1970)

27. Donald, A.S., Yates, A.D., Soh, C.P., Morgan, W.T., Watkins, W.M.: A blood group Sda-active pentasaccharide isolated from Tamm-Horsfall urinary glycoprotein. Biochem. Biophys. Res. Commun. 115, 625-631 (1983)

28. Blanchard, D., Capon, C., Leroy, Y., Cartron, J.P., Fournet, B.: Comparative study of glycophorin a derived O-glycans from human Cad, Sd(a+) and Sd(a-) erythrocytes. Biochem. J. 232, 813$818(1985)$ 
29. Dall'Olio, F., Malagolini, N., Chiricolo, M., Trinchera, M., Harduin-Lepers, A.: The expanding roles of the $\mathrm{Sd}(\mathrm{a}) / \mathrm{Cad}$ carbohydrate antigen and its cognate glycosyltransferase B4GALNT2. Biochim. Biophys. Acta 1840, 443-453 (2014)

30. Shevinsky, L.H., Knowles, B.B., Damjanov, I., Solter, D.: Monoclonal antibody to murine embryos defines a stage-specific embryonic antigen expressed on mouse embryos and human teratocarcinoma cells. Cell 30, 697-705 (1982)

31. Kannagi, R., Nudelman, E., Levery, S.B., Hakomori, S.: A series of human erythrocyte glycosphingolipids reacting to the monoclonal antibody directed to a developmentally regulated antigen SSEA-1. J. Biol. Chem. 257, 14865-14874 (1982)

32. Kannagi, R., Cochran, N.A., Ishigama, F., Hakomori, S., Andrews, P.W., Knowles, B.B., Solter, D.: Stage-specific embryonic antigens (SSEA-3 and -4) are epitopes of a unique globo-series ganglioside isolated from human teratocarcinoma cells. EMBO J. 2, 2355-2361 (1983)

33. Kannagi, R., Levery, S.B., Ishigami, F., Hakomori, S., Shevinsky, L.H., Knowles, B.B., Solter, D.: New globoseries glycosphingolipids in human teratocarcinoma reactive with the monoclonal antibody directed to a developmentally regulated antigen, stage-specific embryonic antigen 3. J. Biol. Chem. 258, 8934-8942 (1983)

34. Wright, A.J., Andrews, P.W.: Surface marker antigens in the characterization of human embryonic stem cells. Stem Cell Res. 3, 3-11 (2009)

35. Zhao, W., Ji, X., Zhang, F., Li, L., Ma, L.: Embryonic stem cell markers. Molecules 17, 6196-6236 (2012)

36. Riekstina, U., Cakstina, I., Parfejevs, V., Hoogduijn, M., Jankovskis, G., Muiznieks, I., Muceniece, R., Ancans, J.: Embryonic stem cell marker pattern in human mesenchymal stem cells derived from bone marrow, adipose tissue, heart and dermis. Stem Cell Rev. 5, 378-386 (2009)
37. Ramirez, J.M., Gerbal-Chaloin, S., Milhavet, O., Qiang, B., Becker, F., Assou, S., Lemaitre, J.M., Hammah, S., De Vos, J.: Benchmarking human pluripotent stem cell markers during differentiation into the three germ layers unveils a striking heterogeneity: all markers are not equal. Stem Cells 29, 1469-1474 (2011)

38. Barone, A., Säljö, K., Benktander, J., Blomqvist, M., Månsson, J.E., Johansson, B.R., Mölne, J., Aspegren, A., Björquist, P., Breimer, M.E., Teneberg, S.: Sialyl-lactotetra: a novel cell surface marker of undifferentiated human pluripotent stem cells. J. Biol. Chem. 289, 18846-18859 (2014)

39. Dick, J.E.: Future prospects for animal models created by transplanting human haematopoietic cells into immune-deficient mice. Res. Immunol. 145, 380-384 (1994)

40. Chang, W.W., Lee, C.H., Lee, P., Lin, J., Hsu, C.W., Hung, J.T., Lin, J.J., Yu, J.C., Shao, L.E., Yu, J., Wong, C.H., Yu, A.L.: Expression of Globo $\mathrm{H}$ and SSEA3 in breast cancer stem cells and the involvement of fucosyl transferases 1 and 2 in Globo $\mathrm{H}$ synthesis. Proc. Natl. Acad. Sci. U. S. A. 105, 11667-11672 (2008)

41. Ye, F., Li, Y., Hu, Y., Zhou, C., Chen, H.: Stage-specific embryonic antigen 4 expression in epithelial ovarian carcinoms. Int. J. Gynecol. Cancer 20, 958-964 (2010)

42. Saito, S., Orikasa, S., Satoh, M., Ohyama, C., Ito, A., Takahashi, T.: Expression of globo-series gangliosides in human renal cell carcinoma. Jpn. J. Cancer Res. 88, 652-659 (1997)

43. Gottschling, S., Jensen, K., Warth, A., Herth, F.J., Thomas, M., Schnabel, P.A., Herpel, E.: Stage-specific embryonic antigen-4 is expressed in basaloid lung cancer and associated with poor prognosis. Eur. Respir. J. 41, 656-663 (2013)

44. Lou, Y.W., Wang, P.Y., Yeh, S.C., Chuang, P.K., Li, S.T., Wu, C.Y., Khoo, K.H., Hsiao, M., Hsu, T.L., Wong, C.H.: Stage-specific embryonic antigen- 4 as a potential therapeutic target in glioblastoma multiforme and other cancers. Proc. Natl. Acad. Sci. U. S. A. 111, 2482-2487 (2014) 